\title{
Total Synthesis of ent-Callilongisin B
}

Akinobu Kamiya, Yuichiro Kawamoto, Toyoharu Kobayashi, and Hisanaka Ito ${ }^{*}$

School of Life Sciences, Tokyo University of Pharmacy and Life Sciences, 1432-1 Horinouchi, Hachioji, Tokyo 192-0392, Japan

itohisa@toyaku.ac.jp

\section{Table of Contents}

Experimental procedure

S2

${ }^{1} \mathrm{H}$ and ${ }^{13} \mathrm{C}$ NMR spectra of new compounds

S16

X-Ray Crystallography data for compound: 17

S35 
General

All reactions involving air- and moisture-sensitive reagents were carried out using standard syringe-septum cap techniques. Unless otherwise noted, all solvents were obtained from commercial suppliers and used without further purification. Routine monitoring of reactions was carried out Merk silica gel 60 F254 TLC plates. Column chromatography was performed on Kanto Chemical Silica Gel 60N (spherical, neutral $60-230 \mu \mathrm{m})$ with the solvents indicated. Melting points were taken on a Yanako MP-S3 micro melting point apparatus and are uncorrected. ${ }^{1} \mathrm{H}$ and ${ }^{13} \mathrm{C}$ NMR sepectra were measured by a spectrometer of Jeol ECZ-400s (400 MHz) or a Bruker AV-500 (500 $\mathrm{MHz}$ ). Chemical shifts were expressed in ppm using $\mathrm{CHCl}_{3}$ (7.26 ppm for ${ }^{1} \mathrm{H} \mathrm{NMR}$, $77.0 \mathrm{ppm}$ for ${ }^{13} \mathrm{C} \mathrm{NMR}$ ) in $\mathrm{CDCl}_{3}$. Measurement of optical rotations was performed with a JASCO P-2200 polarmeter. Infrared spectral measurements were carried out with a JASCO FT/IR-4700 and only noteworthy absorptions were listed. HRMS spectra measured on a Micromass LCT spectrometer. X-ray crystallographic analysis was taken with Burker APEX2 Ultra TXS. Oil bath was used in reactions that require heating and each temperature indicates oil bath temperature. Commercially available chemicals were employed without further purification if not stated otherwise.

((4S)-4-(prop-1-en-2-yl)-7-oxabicyclo[4.1.0]heptan-1-yl)methanol (10)

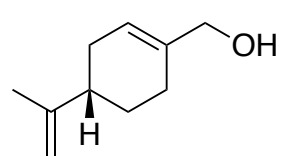

(S)-Perillyl alcohol (9)

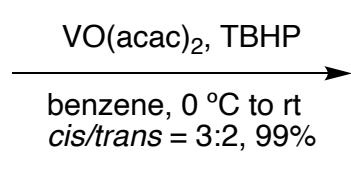

(1)


at room temperature. The reaction mixture was quenched with sat. $\mathrm{NaHSO}_{3}$ aqueous solution at the same temperature, and extracted with AcOEt three times. The combined organic layers were washed with brine, dried over $\mathrm{MgSO}_{4}$, and evaporated under reduced pressure. The resulting residue was purified by column chromatography (hexane-AcOEt, 5:1) to afford 10 (16.3 g, $97.6 \mathrm{mmol}, 99 \%)$ as a colorless oil and a mixture of 3:2 ratio. The cis and trans of compounds $\mathbf{1 0}$ were determined by making a comparison with ${ }^{1} \mathrm{H}$ spectra of the original literature. Those spectra data were identical to previous reports ${ }^{1}$.

${ }^{1} \mathrm{H}$ NMR $\left(400 \mathrm{MHz}, \mathrm{CDCl}_{3}\right) \delta 4.73(1 \mathrm{H}, \mathrm{s}), 4.68(1 \mathrm{H}, \mathrm{s}), 3.76-3.55(1 \mathrm{H}, \mathrm{m}), 3.36-3.29$ $(1 \mathrm{H}, \mathrm{m}), 2.23-2.02(2 \mathrm{H}, \mathrm{m}), 1.92-1.77(2 \mathrm{H}, \mathrm{m}), 1.70(3 \mathrm{H}, \mathrm{s}), 1.68-1.59(2 \mathrm{H}, \mathrm{m})$, $1.48-1.16(1 \mathrm{H}, \mathrm{m})$

1) K. Mori Tetrahedron: Asymmetry 2006, 17, 2133-2142.

(5S)-2-oxo-5-(prop-1-en-2-yl)cyclohexyl acetate (11)

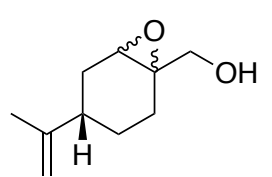

10

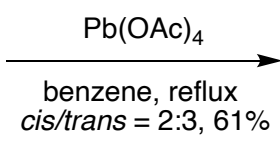

benzene, reflux
cis/trans $=2: 3,61 \%$

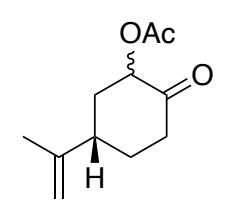

11

To a stirred solution of $\mathbf{1 0}(15.2 \mathrm{~g}, 89.2 \mathrm{mmol})$ in benzene $(180 \mathrm{~mL})$ were added lead tetraacetate $(47.4 \mathrm{~g}, 107 \mathrm{mmol})$ at room temperature and the reaction mixture was refluxed for $2 \mathrm{~h}$. The reaction mixture was quenched with sat. $\mathrm{NaHSO}_{3}$ aqueous solution at room temperature, and extracted with AcOEt three times. The combined organic layers were washed with brine, dried over anhydrous $\mathrm{MgSO}_{4}$, and evaporated under reduced pressure. The resulting residue was purified by column chromatography (hexane-AcOEt, 7:1) to afford $11(10.6 \mathrm{~g}, 54.4 \mathrm{mmol}, 61 \%$ ) as a colorless oil and a mixture of 2:3 ratio. The cis and trans of compounds 11 were determined by making a comparison with ${ }^{1} \mathrm{H}$ spectra of the original literature. Those spectra data were identical to those previous reports ${ }^{2}$.

2) H. Bouanou et al. Tetrahedron Lett. 2011, 52, 4017-4020.

The diastereomeric mixture was separated to isolate the polar diastereomer, and data and spectra show only the polar diastereomer. 
${ }^{1} \mathrm{H}$ NMR $\left(400 \mathrm{MHz}, \mathrm{CDCl}_{3}\right) \delta 5.26(1 \mathrm{H}, \mathrm{ddd}, J=12.9,6.3,0.8 \mathrm{~Hz}), 4.80(1 \mathrm{H}, \mathrm{t}, J=1.4$ Hz), $4.78(1 \mathrm{H}, \mathrm{br}), 2.64-2.40(3 \mathrm{H}, \mathrm{m}), 2.31(1 \mathrm{H}, \mathrm{m}), 2.15(3 \mathrm{H}, \mathrm{s}), 2.11(1 \mathrm{H}, \mathrm{m}), 1.80$ $(1 \mathrm{H}, \mathrm{m}), 1.76(1 \mathrm{H}, \mathrm{m}), 1.62(1 \mathrm{H}, \mathrm{m})$

(5S)-2-oxo-5-(prop-1-en-2-yl)cyclohexyl acetate (S1)

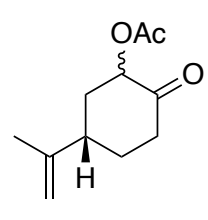

11

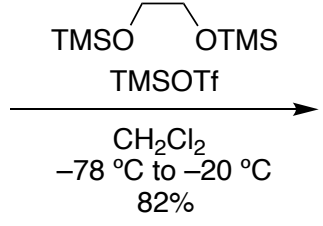

$82 \%$

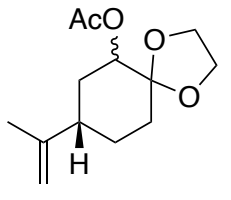

S1

To a stirred solution of 11 (2.53 g, $12.9 \mathrm{mmol})$ in $\mathrm{CH}_{2} \mathrm{Cl}_{2}(14 \mathrm{~mL})$ containing TMSOTf $(0.230 \mathrm{~mL}, 286 \mathrm{mg}, 1.29 \mathrm{mmol})$ were added ethylenedioxybis(trimethylsilane) (3.77 $\mathrm{mL}, 3.18 \mathrm{~g} 15.4 \mathrm{mmol}$ ) at $-78{ }^{\circ} \mathrm{C}$ under $\mathrm{Ar}$ atmosphere. The mixture was stirred at this temperature for $1 \mathrm{~h}$. This mixture was allowed to warm $-20{ }^{\circ} \mathrm{C}$, and quenched with pyridine $(0.260 \mathrm{~mL}, 3.22 \mathrm{mmol})$, poured into sat. $\mathrm{NaHCO}_{3}$ aqueous solution, and extracted with $\mathrm{Et}_{2} \mathrm{O}$ three times. The combined organic layers were washed with brine, dried over anhydrous $\mathrm{MgSO}_{4}$, and evaporated under reduced pressure. The resulting residue was purified by column chromatography (hexane-AcOEt, 10:1) to afford $\mathbf{S 1}$ $(2.58 \mathrm{~g}, 10.7 \mathrm{mmol}, 82 \%)$ as a colorless oil and diastereomeric mixtures.

The diastereomeric mixture was separated to isolate the polar diastereomer, and data and spectra show only the polar diastereomer.

$[\alpha]_{\mathrm{D}}^{25}=+10.4\left(\mathrm{c} 0.59, \mathrm{CHCl}_{3}\right)$; IR (neat) 2941, 1743, 1593, 1240, 1374, 1085, $1057 \mathrm{~cm}^{-}$ 1; ${ }^{1} \mathrm{H}$ NMR (400 MHz, $\left.\mathrm{CDCl}_{3}\right) \delta ; 4.90(1 \mathrm{H}, \mathrm{dd}, J=11.9,4.6 \mathrm{~Hz}), 4.68-4.70(2 \mathrm{H}, \mathrm{m})$, 4.04-4.09 (1H, m), 3.93-3.98 (3H, m), 2.07-2.15 (1H, dddd, $J=12.4,12.4,3.0,3.0 \mathrm{~Hz})$, $2.05(3 \mathrm{H}, \mathrm{s}), 1.91-1.97(1 \mathrm{H}, \mathrm{m}), 1.77-1.81(1 \mathrm{H}, \mathrm{ddd}, J=12.8,2.9,2.9 \mathrm{~Hz}), 1.69(3 \mathrm{H}, \mathrm{s})$, 1.44-1.68 (4H, m); ${ }^{13} \mathrm{C}$ NMR (100 MHz, $\left.\mathrm{CDCl}_{3}\right) \delta 170.3,148.0,109.5,108.2,74.9$, $65.9,65.8,42.8,34.7,34.3,28.3,21.4,20.8$, An extra peak at 76.674 is impurity.; HRMS (ESI-TOF) Calcd for $\mathrm{C}_{13} \mathrm{H}_{20} \mathrm{O}_{4} \mathrm{Na}[\mathrm{M}+\mathrm{Na}]^{+} 263.1254$. Found 263.1250. 

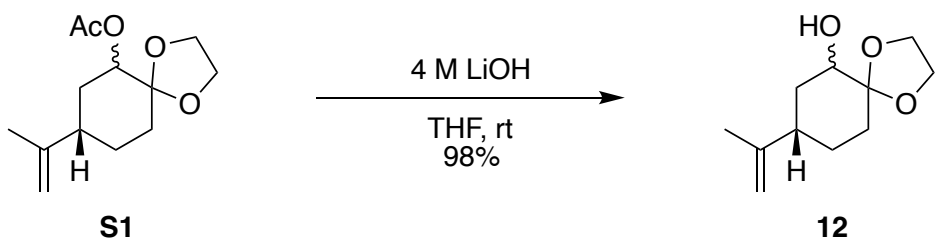

To a stirred solution of $\mathbf{S 1}(4.30 \mathrm{~g}, 18.0 \mathrm{mmol})$ in THF $(25 \mathrm{~mL})$ were added $4 \mathrm{M} \mathrm{LiOH}$ $(90.0 \mathrm{~mL}, 360 \mathrm{mmol})$ at room temperature and the reaction mixture was stirred for $10 \mathrm{~h}$ at the same temperature. The reaction mixture was quenched with sat. $\mathrm{NH}_{4} \mathrm{Cl}$ aqueous solution and extracted with AcOEt three times. The combined organic layers were washed with brine, dried over anhydrous $\mathrm{MgSO}_{4}$, and evaporated under reduced pressure. The resulting residue was purified by column chromatography (hexane-AcOEt, 2:1) to afford 12 (3.48 g, $17.6 \mathrm{mmol}, 98 \%)$ as a colorless oil and diastereomeric mixtures.

The diastereomeric mixture was separated to isolate each diastereomer, and data and spectra show both the polar and less polar diastereomer.

Data for polar diastereomer; $[\alpha]^{25}=-10.9\left(\mathrm{c} 0.72, \mathrm{CHCl}_{3}\right)$; IR (neat) 3476, 2939, 1644, 1434, 1376, 1134, 1104, 1051, $1019 \mathrm{~cm}^{-1} ;{ }^{1} \mathrm{H} \mathrm{NMR}\left(400 \mathrm{MHz}, \mathrm{CDCl}_{3}\right) \delta 4.71(1 \mathrm{H}, \mathrm{s})$, 4.69-4.71 (1H, dd, $J=1.6,1.6 \mathrm{~Hz}), 3.89-4.02(4 \mathrm{H}, \mathrm{m}), 3.65$ (1H, br), 2.35 (1H, dddd, $J$ $=12.4,12.4,3.4,3.4 \mathrm{~Hz}), 2.10-2.17(1 \mathrm{H}, \mathrm{br}), 1.83-2.03(2 \mathrm{H}, \mathrm{m}), 1.71(3 \mathrm{H}, \mathrm{s}), 1.64-$ $1.71(2 \mathrm{H}, \mathrm{m}), 1.41-1.61(2 \mathrm{H}, \mathrm{m}) ;{ }^{13} \mathrm{C} \mathrm{NMR}\left(100 \mathrm{MHz}, \mathrm{CDCl}_{3}\right) \delta 149.3,109.0,108.9$, 69.8, 65.5, 64.4, 37.0, 34.4, 30.0, 28.3, 21.0; HRMS (ESI-TOF) Calcd for $\mathrm{C}_{11} \mathrm{H}_{18} \mathrm{O}_{3}$ $[\mathrm{M}+\mathrm{Na}]^{+}$221.1148. Found 221.1146.

Data for less polar diastereomer; $[\alpha]^{25} \mathrm{D}=-6.56\left(\mathrm{c} 0.65, \mathrm{CHCl}_{3}\right.$ ); IR (neat) 3433, 2940, 1644, 1439, 1276, 1174, 1087, 932, 888; ${ }^{1} \mathrm{H}$ NMR (400 MHz, $\left.\mathrm{CDCl}_{3}\right) \delta 4.71(1 \mathrm{H}, \mathrm{s})$, $4.69(1 \mathrm{H}, \mathrm{dd}, J=1.4,1.4 \mathrm{~Hz}), 3.97-4.11(4 \mathrm{H}, \mathrm{m}), 3.64-3.70(1 \mathrm{H}, \mathrm{ddd}, J=11.9,7.1,4.6$ Hz), 1.95-2.09 (2H, m), 1.80-1.89 (2H, m), $1.71(3 \mathrm{H}, \mathrm{s}), 1.61-1.71(1 \mathrm{H}, \mathrm{m}), 1.38-1.54$ $(2 \mathrm{H}, \mathrm{m}) ;{ }^{13} \mathrm{C} \mathrm{NMR}\left(100 \mathrm{MHz}, \mathrm{CDCl}_{3}\right) \delta 148.5,109.4,109.2,73.0,65.6,65.4,43.0$, 37.3, 33.5, 28.4, 20.8; HRMS (ESI-TOF) Calcd for $\mathrm{C}_{11} \mathrm{H}_{18} \mathrm{O}_{3} \mathrm{Na}[\mathrm{M}+\mathrm{Na}]^{+} 221.1148$. Found 221.1146.

(S)-8-(prop-1-en-2-yl)-1,4-dioxaspiro[4.5]decan-6-one (S2) 


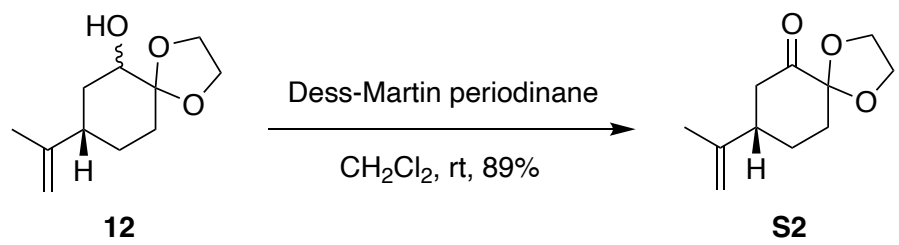

To a stirred solution of 12 (631 mg, $3.18 \mathrm{mmol})$ in $\mathrm{CH}_{2} \mathrm{Cl}_{2}(3 \mathrm{~mL})$ was added DessMartin periodinane $(2.69 \mathrm{~g}, 6.36 \mathrm{mmol})$ at room temperature, and the mixture was stirred for $8 \mathrm{~h}$ at the same temperature. The reaction was quenched with sat. $\mathrm{NaHCO}_{3}$ aqueous solution and extracted with $\mathrm{CHCl}_{3}$ three times. The combined organic layers were washed with brine, dried over anhydrous $\mathrm{MgSO}_{4}$, and evaporated under reduced pressure. The resulting residue was purified by column chromatography (hexane-AcOEt, 5:1) to afford $\mathbf{S 2}(556 \mathrm{mg}, 2.83 \mathrm{mmol}, 89 \%)$ as a colorless oil. $[\alpha]^{25} \mathrm{D}=-27.0$ (c 0.52 , $\mathrm{CHCl}_{3}$ ); IR (neat) 2951, 1731, 1585, 1434, 1197, 1100, 1061, 1024, 938, 894; ${ }^{1} \mathrm{H}$ NMR $\left(400 \mathrm{MHz} \mathrm{CDCl}_{3}\right) \delta 4.77(1 \mathrm{H}, \mathrm{dd}, J=1.4,1.4 \mathrm{~Hz}), 4.74(1 \mathrm{H}, \mathrm{s}), 4.03-4.11(2 \mathrm{H}, \mathrm{m})$, $3.97(1 \mathrm{H}, \mathrm{m}), 3.85(1 \mathrm{H}, \mathrm{m}), 2.70(1 \mathrm{H}, \mathrm{dd}, J=13.5,12.1 \mathrm{~Hz}), 2.47$ (1H, ddd, $J=13.6$, 3.8, 1.7), 2.32-2.41 (1H, m), 2.01-2.11 (1H, m), $1.83-1.93(3 \mathrm{H}, \mathrm{m}), 1.73(3 \mathrm{H}, \mathrm{s}) ;{ }^{13} \mathrm{C}$ NMR (100 MHz, $\left.\mathrm{CDCl}_{3}\right) \delta 205.9,146.6,110.6,106.4,65.9,64.7,45.1,44.3,35.0,27.6$, 20.5; HRMS (ESI-TOF) Calcd for $\mathrm{C}_{11} \mathrm{H}_{16} \mathrm{O}_{3} \mathrm{Na}[\mathrm{M}+\mathrm{Na}]^{+}$219.0992. Found 219.0995.

(8S)-7-methyl-8-(prop-1-en-2-yl)-1,4-dioxaspiro[4.5]decan-6-one (8)<smiles>C=C(C)[C@H]1CCC2(OCCO2)C(=O)C1</smiles>

S2

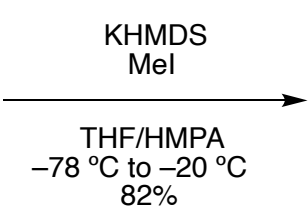

$82 \%$

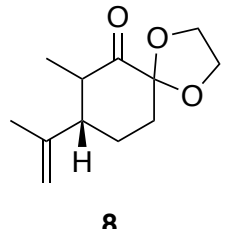

To a stirred solution of $\mathbf{S 2}(1.00 \mathrm{~g}, 5.10 \mathrm{mmol})$ in THF $(5 \mathrm{~mL})$ and HMPA $(1 \mathrm{~mL})$ were added dropwise KHMDS (0.5 $\mathrm{M}$ in toluene, $20.4 \mathrm{~mL}, 10.2 \mathrm{mmol})$ at $-78{ }^{\circ} \mathrm{C}$ under $\mathrm{Ar}$ atmosphere, and the reaction mixture was stirred for $1.5 \mathrm{~h}$ at the same temperature. To this mixture was added dropwise $\mathrm{MeI}(0.950 \mathrm{~mL}, 2.17 \mathrm{~g}, 15.3 \mathrm{mmol})$ at $-78^{\circ} \mathrm{C}$, and the mixture was allowed to warm to $-20^{\circ} \mathrm{C}$ over a period of $2 \mathrm{~h}$. The reaction mixture was quenched with sat. $\mathrm{NH}_{4} \mathrm{Cl}$ aqueous solution, and extracted with AcOEt three times. The combined organic layers were washed with brine, dried over anhydrous $\mathrm{MgSO}_{4}$, and evaporated under reduced pressure. The resulting residue was purified by column 
chromatography (hexane-AcOEt) to afford $8(880 \mathrm{mg}, 4.19 \mathrm{mmol})$ as a colorless oil. $[\alpha]^{25_{\mathrm{D}}}=-46.6\left(\mathrm{c} 0.80, \mathrm{CHCl}_{3}\right)$; IR (neat) 2970, 2936, 2894, 1729, 1204, 1184, 1028, 970, 921, 894; ${ }^{1} \mathrm{H}$ NMR (400 MHz, $\left.\mathrm{CDCl}_{3}\right) \delta 4.78(1 \mathrm{H}, \mathrm{s}), 4.74(1 \mathrm{H}, \mathrm{s}), 4.03-4.09(2 \mathrm{H}, \mathrm{m})$, 3.92-4.00 (1H, m), 3.79-3.88 (1H, m), 2.73-2.82 (1H, m), 2.34-2.47 (1H, m), 1.99$2.09(2 \mathrm{H}, \mathrm{m}), 1.80-1.91(2 \mathrm{H}, \mathrm{m}), 1.69(3 \mathrm{H}, \mathrm{s}), 0.92(3 \mathrm{H}, \mathrm{d}, J=6.4 \mathrm{~Hz}) ;{ }^{3} \mathrm{C}$ NMR $(100$ $\left.\mathrm{MHz}, \mathrm{CDCl}_{3}\right) \delta$ 207.4, 145.5, 112.7, 106.7, 65.8, 64.4, 53.6, 44.8, 35.6, 27.9, 17.8, 11.6; HRMS (ESI-TOF) Calcd for $\mathrm{C}_{12} \mathrm{H}_{18} \mathrm{O}_{3} \mathrm{Na}[\mathrm{M}+\mathrm{Na}]^{+} 233.1148$. Found 233.1145.

$N, N$-dimethyl-3-((7R,8R)-7-methyl-6-oxo-8-(prop-1-en-2-yl)-1,4-dioxaspiro[4.5]decan7-yl)propenamide (7)
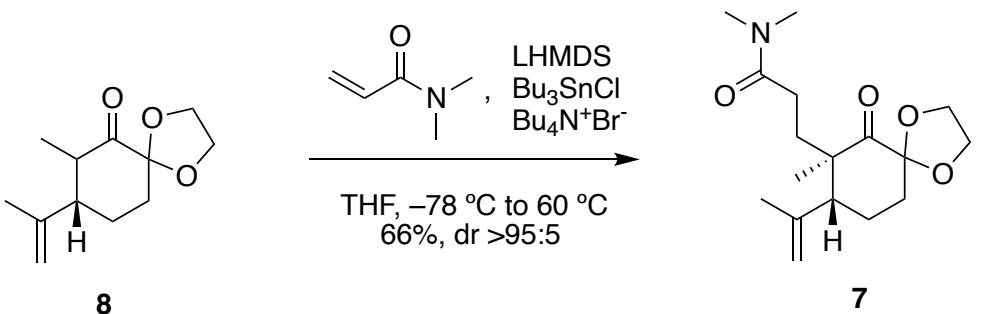

To a stirred solution of $8(1.70 \mathrm{~g}, 8.09 \mathrm{mmol})$ in THF $(17 \mathrm{~mL})$ were added dropwise LHMDS (1.0 M in THF, $24.0 \mathrm{~mL}, 24.2 \mathrm{mmol}$ ) at $-78{ }^{\circ} \mathrm{C}$ under $\mathrm{Ar}$, and the reaction mixture was stirred for $1.5 \mathrm{~h}$ at the same temperature. To this mixture was added dropwise $\mathrm{Bu}_{3} \mathrm{SnCl}(6.57 \mathrm{~mL}, 7.88 \mathrm{~g}, 24.2 \mathrm{mmol})$ and $\mathrm{Bu}_{4} \mathrm{~N}^{+} \mathrm{Br}^{-}(260 \mathrm{mg}, 0.809 \mathrm{mmol}$ ) in THF $(1 \mathrm{~mL})$, and stirred for $0.5 \mathrm{~h}$. To the reaction mixture was added $N, N$-dimethyl acrylamide $(3.33 \mathrm{~mL}, 3.19 \mathrm{~g}, 37.1 \mathrm{mmol})$ at $-78{ }^{\circ} \mathrm{C}$, and the mixture was allowed to warm to $60{ }^{\circ} \mathrm{C}$. After the reaction mixture was stirred for 3 days, quenched with sat. $\mathrm{NH}_{4} \mathrm{Cl}$ aqueous solution and extracted with $\mathrm{Et}_{2} \mathrm{O}$ three times. The combined organic layers were washed with brine, dried over $\mathrm{MgSO}_{4}$, and evaporated under reduced pressure. The resulting residue was purified by column chromatography (hexane-AcOEt, $1: 1)$ to afford $7(1.64 \mathrm{~g}, 5.33 \mathrm{mmol}, 66 \%)$ as an orange oil. $[\alpha]^{25}=-31.1(\mathrm{c} 1.35$, $\mathrm{CHCl}_{3}$ ); IR (neat) 1714, 1645, 1496, 1455, 1397, 1207, 1143, 1026, 967; ${ }^{1} \mathrm{H}$ NMR (400 $\left.\mathrm{MHz} \mathrm{CDCl}_{3}\right) \delta 4.94(1 \mathrm{H}, \mathrm{s}), 4.69(1 \mathrm{H}, \mathrm{s}), 4.09-3.98(3 \mathrm{H}, \mathrm{m}), 3.90-3.82(1 \mathrm{H}, \mathrm{m}), 3.04$ $(3 \mathrm{H}, \mathrm{s}), 2.91(3 \mathrm{H}, \mathrm{s}), 2.42-2.39(1 \mathrm{H}, \mathrm{m}), 2.20-2.35(2 \mathrm{H}, \mathrm{m}), 2.16-2.20(2 \mathrm{H}, \mathrm{m})$, 1.96-1.82 (4H, m), 1,78 (3H, s), $1.16(3 \mathrm{H}, \mathrm{s}) ;{ }^{13} \mathrm{C} \mathrm{NMR}\left(100 \mathrm{MHz}, \mathrm{CDCl}_{3}\right) \delta 209.7$, 173.1, 144.2, 114.9, 106.2, 65.9, 64.9, 51.9, 49.9, 37.4, 35.4, 34.4, 32.8, 28.8, 24.1, 23.8, 20.7; HRMS (ESI-TOF) Calcd for $\mathrm{C}_{17} \mathrm{H}_{27} \mathrm{NO}_{4} \mathrm{Na}[\mathrm{M}+\mathrm{Na}]^{+} 332.1832$. Found 332.1833. 
3-((7R,8R)-6-hydroxy-7-methyl-8-(prop-1-en-2-yl)-6-((trimethylsilyl)methyl)-1,4-dioxa spiro[4.5]decan-7-yl)- $N, N$-dimethylpropanamide (S3)

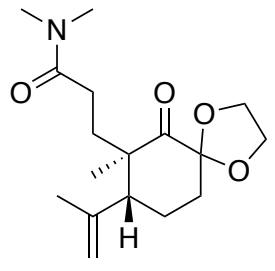

7

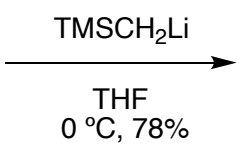

$0^{\circ} \mathrm{C}, 78 \%$

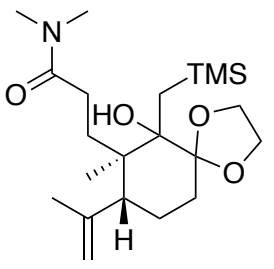

S3

To a stirred solution of 7 (970 $\mathrm{mg}, 3.13 \mathrm{mmol})$ in THF $(1.5 \mathrm{~mL})$ was added dropwise $\mathrm{TMSCH}_{2} \mathrm{Li}(1.0 \mathrm{M}$ in pentane, $4.70 \mathrm{~mL}, 4.70 \mathrm{mmol})$ at $0{ }^{\circ} \mathrm{C}$, and the mixture was stirred for $20 \mathrm{~min}$ at $0{ }^{\circ} \mathrm{C}$. The reaction was quenched with sat. $\mathrm{NH}_{4} \mathrm{Cl}$ aqueous solution, and extracted with AcOEt three times. The combined organic layers were washed with brine, dried over $\mathrm{MgSO}_{4}$, and evaporated under reduced pressure. The resulting residue was purified by column chromatography (hexane-AcOEt, 1:1) to afford S3 (976 mg, $2.45 \mathrm{mmol}, 78 \%)$ as a colorless oil. $[\alpha]^{25}{ }_{\mathrm{D}}=-1.09\left(\mathrm{c} 0.45 \mathrm{CHCl}_{3}\right)$; IR (neat) 1645, 1396, 1350, 1241, 1144, 1105, 1080, 861, 837; ${ }^{1} \mathrm{H}$ NMR (400 MHz, $\left.\mathrm{CDCl}_{3}\right) \delta 4.86(1 \mathrm{H}, \mathrm{s})$, 4,73 (1H, s), 4.12-4.07 (1H, m), 3.94-3.76 (3H, m), $2.97(3 \mathrm{H}, \mathrm{s}), 2.91(3 \mathrm{H}, \mathrm{s}), 2.58(1 \mathrm{H}$, ddd, $J=15.8 \mathrm{~Hz}, 12.4 \mathrm{~Hz}, 5.0 \mathrm{~Hz}), 2.26-2.21(2 \mathrm{H}, \mathrm{m}), 1.97-1.89(1 \mathrm{H}, \mathrm{m}), 1.85-1.53$ $(7 \mathrm{H}, \mathrm{m}), 1.34-1.29(1 \mathrm{H}, \mathrm{m}), 1.15(2 \mathrm{H}, \mathrm{m}), 1.03(3 \mathrm{H}, \mathrm{s}), 0.05(9 \mathrm{H}, \mathrm{s}) ;{ }^{13} \mathrm{C}$ NMR $(100$ $\left.\mathrm{MHz}, \mathrm{CDCl}_{3}\right) \delta 174.1,147.4,113.5,112.6,81.0,65.3,64.0,50.0,46.0,37.0,35.1,33.4$, 31.5, 29.1, 25.4, 24.8, 22.7, 13.5, 1.2 (3C); HRMS (ESI-TOF) Calcd for $\mathrm{C}_{21} \mathrm{H}_{39} \mathrm{NO}_{4} \mathrm{SiNa}[\mathrm{M}+\mathrm{Na}]^{+}$420.2541. Found 420.2539.

$N, N$-dimethyl-3-((7R,8R)-7-methyl-6-methylene-8-(prop-1-en-2-yl)-1,4-dioxaspiro[4.5] decan-7-yl)propenamide (13)<smiles>C=C(C)[C@H]1CCC2(OCCO2)[C@H](C)[C@@H]1CCC(=O)N(C)C</smiles>

S3

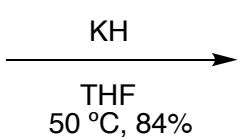

$50^{\circ} \mathrm{C}, 84 \%$

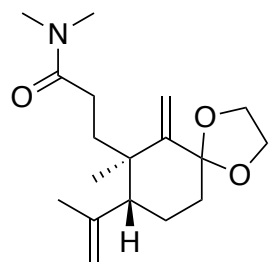

13

To a stirred suspension of KH (30\% in mineral oil, $388 \mathrm{mg}, 2.94 \mathrm{mmol})$ in THF (10 $\mathrm{mL})$ was added dropwise a solution of $\mathbf{S 3}(976 \mathrm{mg}, 2.45 \mathrm{mmol})$ in THF $(1.0 \mathrm{~mL})$ at $0{ }^{\circ} \mathrm{C}$ 
under Ar, and the mixture was stirred for $2 \mathrm{~h}$ at $50^{\circ} \mathrm{C}$. The reaction mixture was quenched with THF/MeOH $(10: 1)$ at $0{ }^{\circ} \mathrm{C}$, and extracted with $\mathrm{Et}_{2} \mathrm{O}$ three times. The combined organic layers were washed with brine, dried over $\mathrm{MgSO}_{4}$, and evaporated under reduced pressure. The resulting residue was purified by column chromatography (hexane-AcOEt, 1:1) to afford $13(629 \mathrm{mg}, 2.05 \mathrm{mmol}, 84 \%)$ as a colorless oil. $\left[\alpha{ }^{25} \mathrm{D}=\right.$ +11.9 (c $1.75 \mathrm{CHCl}_{3}$ ); IR (neat) 1644, 1494, 1455, 1396, 1155, 1089, 1064, 916; ${ }^{1} \mathrm{H}$ NMR (400 MHz, $\left.\mathrm{CDCl}_{3}\right) \delta 5.40(1 \mathrm{H}, \mathrm{d}, J=1.4 \mathrm{~Hz}), 4.95(1 \mathrm{H}, \mathrm{d}, J=1.3 \mathrm{~Hz}), 4.85-4.84$ $(1 \mathrm{H}, \mathrm{m}), 4.74(1 \mathrm{H}, \mathrm{br}), 3.97-3.84(4 \mathrm{H}, \mathrm{m}), 2.99(3 \mathrm{H}, \mathrm{s}), 2.93(3 \mathrm{H}, \mathrm{s}), 2.26-2.21(3 \mathrm{H}, \mathrm{m})$, 2.13-2.09 (1H, m), 2.06-1.96 (2H, m), $1.73(3 \mathrm{H}, \mathrm{s}), 1.71-1.62(3 \mathrm{H}, \mathrm{m}), 1.11(3 \mathrm{H}, \mathrm{s}) ;{ }^{13} \mathrm{C}$ NMR $\left(100 \mathrm{MHz}, \mathrm{CDCl}_{3}\right) \delta 173.6,149.8,146.2,112.9,110.8,108.2,64.3(2 \mathrm{C}), 51.1$, 42.7, 37.0, 35.3, 34.8, 33.5, 28.5, 24.7, 23.4, 23.3; HRMS (ESI-TOF) Calcd for $\mathrm{C}_{18} \mathrm{H}_{29} \mathrm{NO}_{3} \mathrm{Na}[\mathrm{M}+\mathrm{Na}]^{+} 330.2040$. Found 330.2041.

$N, N$-dimethyl-3-((1R,6R)-1-methyl-2-methylene-3-oxo-6-(prop-1-en-2-yl)cyclohexyl)pr opanamide (14)

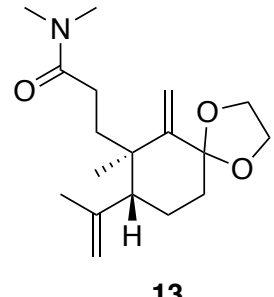

13

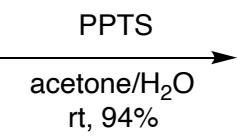

rt, $94 \%$

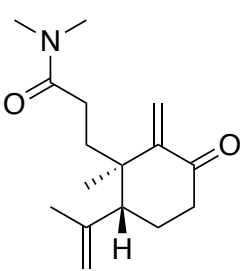

14

To a stirred solution of $13(400 \mathrm{mg}, 1.30 \mathrm{mmol})$ in acetone $(10 \mathrm{~mL})$ and $\mathrm{H}_{2} \mathrm{O}(0.5 \mathrm{~mL})$ was added PPTS (163 mg, $0.651 \mathrm{mmol})$ at room temperature, and the mixture was stirred for $20 \mathrm{~h}$ at the same temperature. The reaction mixture was quenched with sat. $\mathrm{NaHCO}_{3}$ aqueous solution at the same temperature and extracted with AcOEt three times. The combined organic layers were washed with brine, dried over $\mathrm{MgSO}_{4}$, and evaporated under reduced pressure. The resulting residue was purified by column chromatography (hexane-AcOEt, 1:1) to afford 14 (321 mg, $1.22 \mathrm{mmol}, 94 \%)$ as a colorless oil. $[\alpha]^{25}{ }_{\mathrm{D}}=-14.9$ (c $0.48, \mathrm{CHCl}_{3}$ ); IR (neat); 1691, 1644, 1457, 1399, 1264, 1138, 1089, 949, 896; ${ }^{1} \mathrm{H}$ NMR (400 MHz, $\left.\mathrm{CDCl}_{3}\right) \delta 5.89(1 \mathrm{H}, \mathrm{s}), 5.18(1 \mathrm{H}, \mathrm{s})$, 4.91-4.90 (1H, m), 4.78 (1H, br), 2.93-2.91 (6H, m), $2.62(1 \mathrm{H}, \mathrm{ddd}, J=17.3 \mathrm{~Hz}, 8.9 \mathrm{~Hz}$, $7.1 \mathrm{~Hz}), 2.47-2.40(1 \mathrm{H}, \mathrm{m}), 2.32(1 \mathrm{H}, \mathrm{dd}, J=5.3 \mathrm{~Hz}, 5.3 \mathrm{~Hz}), 2.27-2.11(3 \mathrm{H}, \mathrm{m})$, 1.93-1.75 (3H, m), $1.78(3 \mathrm{H}, \mathrm{s}), 1.13(3 \mathrm{H}, \mathrm{s}) ;{ }^{13} \mathrm{C} \mathrm{NMR}\left(100 \mathrm{MHz}, \mathrm{CDCl}_{3}\right) \delta 203.4$, 
172.6, 152.7, 145.7, 119.0, 113.5, 49.5, 43.1, 37.3, 37.1, 35.4, 35.2 27.6, 24.3, 23.6, 22.3; HRMS (ESI-TOF) Calcd for $\mathrm{C}_{16} \mathrm{H}_{25} \mathrm{NO}_{2} \mathrm{Na}[\mathrm{M}+\mathrm{Na}]^{+}$286.1778. Found 286.1774.

3-((1R,6R)-2-(but-3-en-1-yl)-1-methyl-3-oxo-6-(prop-1-en-2-yl)cyclohexyl)- $N, N$-dimet hylpropanamide (S4)

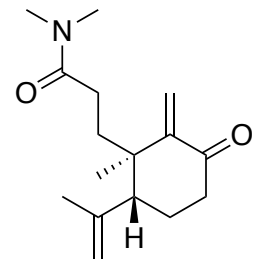

14

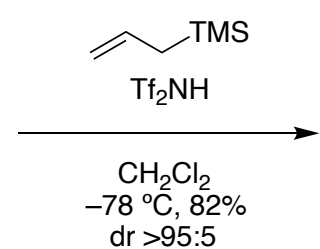

$\mathrm{dr}>95: 5$

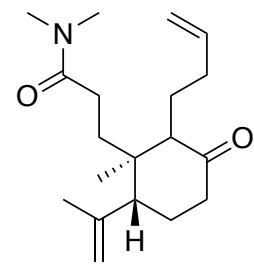

S4

To a stirred solution of $14(100 \mathrm{mg}, 0.380 \mathrm{mmol})$ in $\mathrm{CH}_{2} \mathrm{Cl}_{2}(1.5 \mathrm{~mL})$ were added $\mathrm{Tf}_{2} \mathrm{NH}(210 \mathrm{mg}, 0.760 \mathrm{mmol})$ at $-78{ }^{\circ} \mathrm{C}$ under $\mathrm{Ar}$ and allyltrimethylsilane $(0.120 \mathrm{~mL}$, $87.0 \mathrm{mg}, 0.760 \mathrm{mmol}$ ) at the same temperature. After stirred for $1 \mathrm{~h}$ at the same temperature, the reaction mixture was quenched with sat. $\mathrm{NaHCO}_{3}$ aqueous solution and extracted with $\mathrm{CHCl}_{3}$ three times. The combined organic layers were washed with brine, dried over $\mathrm{MgSO}_{4}$, and evaporated under reduced pressure. The resulting residue was purified by column chromatography (hexane-AcOEt, 1:1) to afford $\mathbf{S 4}$ (95.0 mg, 0.311 mmol, 82\%) as a colorless oil. $[\alpha]_{\mathrm{D}}^{25}=+20.0\left(\mathrm{c} 0.95, \mathrm{CHCl}_{3}\right)$; IR (neat); 3079, 1708, 1641, 1450, 1398, 1262, 1143, 1101, 903; ${ }^{1} \mathrm{H}$ NMR (400 MHz, $\left.\mathrm{CDCl}_{3}\right) \delta 5.73(1 \mathrm{H}$, dddd $J=16.9 \mathrm{~Hz}, 13.9 \mathrm{~Hz}, 7.8 \mathrm{~Hz}, 6.0 \mathrm{~Hz}), 4.97-4.94(2 \mathrm{H}, \mathrm{m}), 4.90(1 \mathrm{H}, \mathrm{s}), 4.76(1 \mathrm{H}$, s), $3.00(3 \mathrm{H}, \mathrm{br}), 2.94(3 \mathrm{H}, \mathrm{br}), 2.60(1 \mathrm{H}, \mathrm{dd} J=12.8 \mathrm{~Hz}, 4.1 \mathrm{~Hz}), 2.49-2.27(4 \mathrm{H}, \mathrm{m})$, 2.29-2.13 (2H, m), 2.04-1.57 (7H, m), 1.75 (3H, s), $0.755(3 \mathrm{H}, \mathrm{s}) ;{ }^{13} \mathrm{C}$ NMR (100 MHz, $\left.\mathrm{CDCl}_{3}\right) \delta 211.6,172.4,146.0,138.6,115.3,114.4,55.9,50.2,44.7,42.2,37.1,35.6$, 32.8 (2C), 28.9, 26.9, 23.3, 21.3, 17.8; HRMS (ESI-TOF) Calcd for $\mathrm{C}_{16} \mathrm{H}_{25} \mathrm{NO}_{2} \mathrm{Na}$ $[\mathrm{M}+\mathrm{Na}]^{+} 286.1778$. Found 286.1774.

\footnotetext{
$N, N$-dimethyl-3-((1R,6R)-1-methyl-3-oxo-2-(3-oxobutyl) -6-(prop-1-en-2-yl)cyclohexyl)propenamide (6)
} 


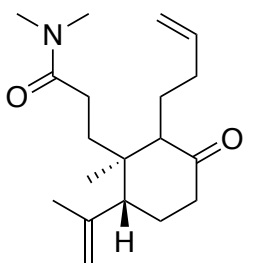

S4

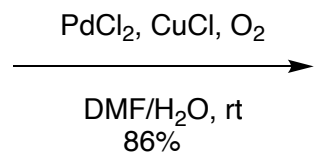

$86 \%$

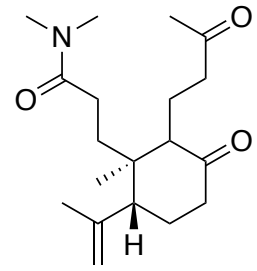

6

To a stirred suspension of $\mathrm{PdCl}_{2}(6.00 \mathrm{mg}, 0.0330 \mathrm{mmol})$ and $\mathrm{CuCl}(33.0 \mathrm{mg}, 0.336$ mmol) in DMF $(5 \mathrm{~mL})$ and $\mathrm{H}_{2} \mathrm{O}(0.5 \mathrm{~mL})$ was bubbled oxygen at room temperature for $1 \mathrm{~h}$. To the mixture was added a solution of $\mathbf{S 4}(102 \mathrm{mg}, 0.336 \mathrm{mmol})$ in DMF (1 mL), and stirred for $15 \mathrm{~h}$ at the same temperature. The reaction mixture was quenched with sat. $\mathrm{NaHCO}_{3}$ aqueous solution and extracted with AcOEt three times. The combined organic layers were washed with brine, dried over anhydrous $\mathrm{MgSO}_{4}$, and evaporated under reduced pressure. The resulting residue was purified by column chromatography $\left(\mathrm{CHCl}_{3}\right.$-AcOEt, $\left.1: 1\right)$ to afford $6(93.0 \mathrm{mg}, 0.289 \mathrm{mmol}, 86 \%)$ as a colorless oil. $[\alpha]^{25}{ }_{\mathrm{D}}=$ -5.12 (c 0.65, MeOH); IR (neat); 1710, 1639, 1399, 1361, 1258, 1163, 1097, 895; ${ }^{1} \mathrm{H}$ NMR (400 MHz, $\left.\mathrm{CDCl}_{3}\right) \delta 4.92(1 \mathrm{H}, \mathrm{s}), 4.77(1 \mathrm{H}, \mathrm{s}), 3.11(3 \mathrm{H}, \mathrm{s}), 2.94(3 \mathrm{H}, \mathrm{s})$, 2.80-2.72 (1H, m), 2.64-2.57 (2H, m), 2.47-2.34 (4H, m), $2.07(3 \mathrm{H}, \mathrm{s}), 2.06-1.94(1 \mathrm{H}$, m), 1.91-1.84 (1H, m), 1.80-1.51 (5H, m), $1.79(3 \mathrm{H}, \mathrm{s}), 0.79(3 \mathrm{H}, \mathrm{s}) ;{ }^{13} \mathrm{C}$ NMR $(100$ $\left.\mathrm{MHz}, \mathrm{CDCl}_{3}\right) \delta 212.4,209.4,172.8,145.8,114.4,55.6,50.0,45.0,42.3,41.5,37.2$, 35.4, 32.9, 30.0, 29.0, 26.7, 23.5, 17.5, 16.4; HRMS (ESI-TOF) Calcd for $\mathrm{C}_{19} \mathrm{H}_{31} \mathrm{NO}_{3} \mathrm{Na}[\mathrm{M}+\mathrm{Na}]^{+}$344.2196. Found 344.2198.

3-((1R,2R)-6-hydroxy-1-methyl-2-(prop-1-en-2-yl)-1,2,3,4-tetrahydronaphthalen-1-yl)$N, N$-dimethylpropanamide (5)
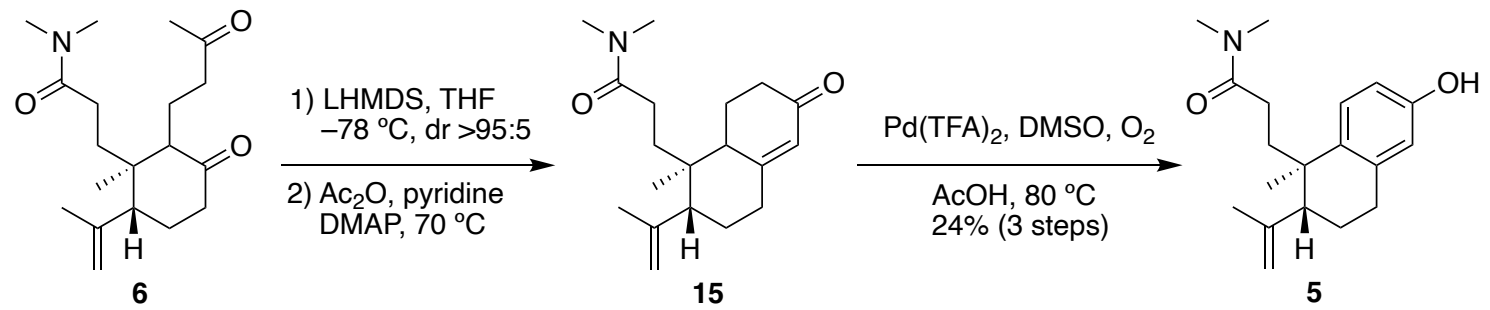

To a stirred solution of $6(75.0 \mathrm{mg}, 0.233 \mathrm{mmol})$ in THF $(1 \mathrm{~mL})$ was added dropwise LHMDS (1.0 M in THF, $0.350 \mathrm{~mL}, 0.350 \mathrm{mmol})$ at $-78{ }^{\circ} \mathrm{C}$ under Ar atmosphere, and the reaction mixture was stirred for $6 \mathrm{~h}$ at the same temperature. The reaction mixture 
was quenched with sat. $\mathrm{NH}_{4} \mathrm{Cl}$ aqueous solution at the same temperature and extracted with AcOEt three times. The combined organic layers were washed with brine, dried over anhydrous $\mathrm{MgSO}_{4}$, and evaporated under reduced pressure. The crude product was dissolved in $\mathrm{Ac}_{2} \mathrm{O}(0.200 \mathrm{~mL}, 2.33 \mathrm{mmol})$ and pyridine $(0.180 \mathrm{~mL}, 2.33 \mathrm{mmol})$ at room temperature, and to the mixture was added DMAP (284 mg, $2.33 \mathrm{mmol})$. The solution was heated at $70{ }^{\circ} \mathrm{C}$ for $0.5 \mathrm{~h}$. The reaction mixture was quenched with $1 \mathrm{M} \mathrm{HCl}(0.2$ $\mathrm{ml}$ ) at room temperature and extracted with $\mathrm{Et}_{2} \mathrm{O}$ three times. The combined organic layers were washed with brine, dried over anhydrous $\mathrm{MgSO}_{4}$, and evaporated under reduced pressure. The resulting residue was purified by column chromatography $\left(\mathrm{CHCl}_{3}\right.$-AcOEt, 1:1) to afford $\mathbf{1 5}$ including an inseparable unknown material. To a stirred suspension of Pd(TFA) 2 ( $7.50 \mathrm{mg}, 0.0230 \mathrm{mmol})$ in DMSO $(0.160 \mathrm{~mL}, 2.33$ mmol) was added a solution of $\mathbf{1 5}$ in $\mathrm{AcOH}(0.5 \mathrm{~mL})$ under $\mathrm{O}_{2}$ atmosphere. After stirred for $6 \mathrm{~h}$ at $80^{\circ} \mathrm{C}$, the reaction mixture was quenched with sat. $\mathrm{NaHCO}_{3}$ aqueous solution at $0{ }^{\circ} \mathrm{C}$ and extracted with $\mathrm{CHCl}_{3}$ three times. The combined organic layers were washed with brine, dried over $\mathrm{MgSO}_{4}$, and evaporated under reduced pressure. The resulting residue was purified by column chromatography $\left(\mathrm{CHCl}_{3}-\mathrm{AcOEt}, 1: 5\right)$ to afford $5(16.0 \mathrm{mg}, 0.0550 \mathrm{mmol}, 24 \%)$ as an orange oil. $[\alpha]^{25} \mathrm{D}=-26.2\left(\mathrm{c} 0.40, \mathrm{CHCl}_{3}\right)$; IR (neat); 3433, 2930, 1625, 1499, 1461, 1402, 753; ${ }^{1} \mathrm{H}$ NMR (400 MHz, $\left.\mathrm{CDCl}_{3}\right) \delta 7.14$ $(1 \mathrm{H}, \mathrm{d}, J=8.7 \mathrm{~Hz}), 6.67(1 \mathrm{H}, \mathrm{dd}, J=8.2 \mathrm{~Hz}, 2.3 \mathrm{~Hz}), 6.51(1 \mathrm{H}, \mathrm{br}), 4.92(1 \mathrm{H}, \mathrm{br}), 4.70$ $(1 \mathrm{H}, \mathrm{br}), 2.89(3 \mathrm{H}, \mathrm{s}), 2.85(3 \mathrm{H}, \mathrm{s}), 2.74-2.72(2 \mathrm{H}, \mathrm{m}), 2.42(1 \mathrm{H}, \mathrm{dd}, J=11.4 \mathrm{~Hz}, 2.3$ $\mathrm{Hz}), 2.28-2.20(1 \mathrm{H}, \mathrm{m}), 2.11-2.08(2 \mathrm{H}, \mathrm{m}), 1.95-1.83(3 \mathrm{H}, \mathrm{m}), 1.78(3 \mathrm{H}, \mathrm{s}), 1.19(3 \mathrm{H}$, s); ${ }^{13} \mathrm{C} \mathrm{NMR}\left(100 \mathrm{MHz}, \mathrm{CDCl}_{3}\right) \delta 173.6,153.4,146.8,138.4,135.4,127.9,114.8$, 114.0, 113.7, 47.5, 40.5, 37.3, 35.5, 35.0, 30.3, 28.4, 28.0, 24.6, 22.8; HRMS (ESITOF) Calcd for $\mathrm{C}_{19} \mathrm{H}_{27} \mathrm{NO}_{2} \mathrm{Na}[\mathrm{M}+\mathrm{Na}]^{+}$324.1934. Found 324.1942.

(4aR,5R,11aS)-4a-methyl-5-(prop-1-en-2-yl)-3,4,4a,5,6,7-hexahydrobenzo[ $i$ ] chromene2,9-dione (16)
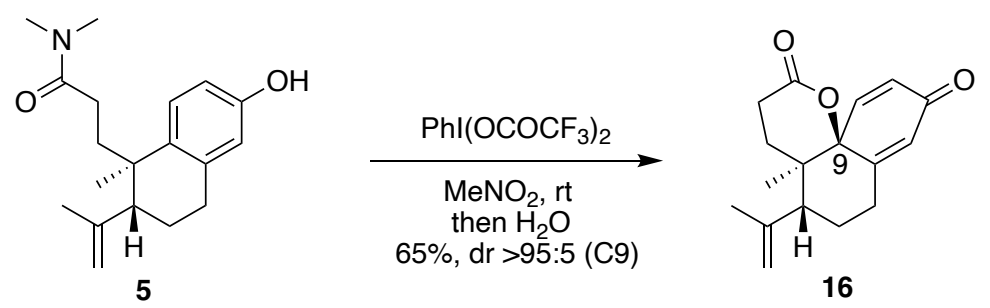
To a stirred solution of $5(50.0 \mathrm{mg}, 0.166 \mathrm{mmol})$ in $\mathrm{MeNO}_{2}(1.2 \mathrm{~mL})$ was added a solution of $\mathrm{PhI}\left(\mathrm{OCOCF}_{3}\right)(86.0 \mathrm{mg}, 0.199 \mathrm{mmol})$ in $\mathrm{MeNO}_{2}(0.3 \mathrm{~mL})$ at room temperature under Ar. The reaction mixture was stirred at the same temperature for $15 \mathrm{~h}$, diluted with water $(0.5 \mathrm{~mL})$ and stirred for $10 \mathrm{~min}$, and extracted with $\mathrm{CHCl}_{3}$ three times. The combined organic layers were washed with brine, dried over $\mathrm{MgSO}_{4}$, and evaporated under reduced pressure. The resulting residue was purified by column chromatography (hexane-AcOE, 3:1) to afford $16(29.0 \mathrm{mg}, 0.107 \mathrm{mmol}, 65 \%)$ as a colorless oil. $[\alpha]^{25}=-6.71$ (c $0.42, \mathrm{CHCl}_{3}$ ); IR (neat); 1741, 1673, 1641, 1615, 1214, 1102, 1041, 996, 896, 756; ${ }^{1} \mathrm{H}$ NMR $\left(400 \mathrm{MHz}, \mathrm{CDCl}_{3}\right) \delta 6.89(1 \mathrm{H}, \mathrm{d}, J=10.0 \mathrm{~Hz})$, $6.33(1 \mathrm{H}, \mathrm{dd}, J=10.0 \mathrm{~Hz}, 1.8 \mathrm{~Hz}), 6.16(1 \mathrm{H}, \mathrm{br}), 5.02(1 \mathrm{H}, \mathrm{br}), 4.82(1 \mathrm{H}, \mathrm{br}), 2.89-2.70$ $(4 \mathrm{H}, \mathrm{m}), 2.44-2.39(1 \mathrm{H}, \mathrm{m}), 2.04-1.79(4 \mathrm{H}, \mathrm{m}), 1.77(3 \mathrm{H}, \mathrm{s}), 0.88(3 \mathrm{H}, \mathrm{s}) ;{ }^{13} \mathrm{C} \mathrm{NMR}$ $\left(100 \mathrm{MHz}, \mathrm{CDCl}_{3}\right) \delta 197.2,182.3,169.0,156.4,155.9,143.4,138.5,128.6,95.1,56.3$, 53.4, 44.1, 40.9, 40.3, 38.3, 35.6, 29.8; HRMS (ESI-TOF) Calcd for $\mathrm{C}_{17} \mathrm{H}_{20} \mathrm{O}_{3} \mathrm{Na}$ $[\mathrm{M}+\mathrm{Na}]^{+}$295.1305. Found 295.1305.

(4aR,5R,11aS)-4a-methyl-5-(prop-1-en-2-yl)-3,4,4a,5,6,7,10,11-octahydrobenzo $[i] \operatorname{chro}$ mene-2,9-dione (17)
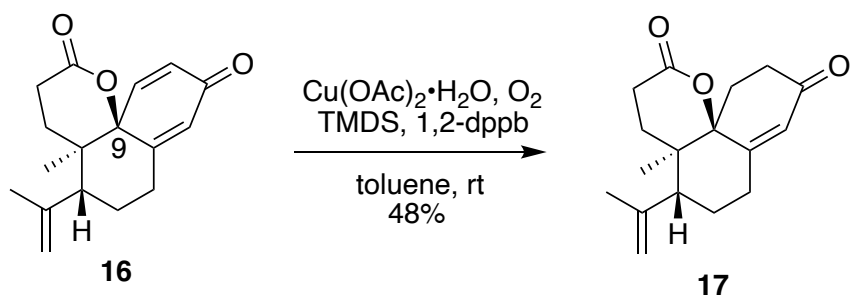

To a stirred suspension of $\mathrm{Cu}(\mathrm{OAc})_{2} \bullet \mathrm{H}_{2} \mathrm{O}(72.0 \mathrm{mg}, 0.364 \mathrm{mmol})$ in toluene $(1.0 \mathrm{~mL})$ was added 1,2-dppb (3.20 mg, $0.199 \mathrm{mmol})$ at room temperature under Ar atmosphere. The suspension was stirred for $10 \mathrm{~min}$ at the same temperature, TMDS $(80.0 \mu \mathrm{L}, 0.459$ mmol) was added, and the atmosphere was exchanged for $\mathrm{O}_{2}$ atmosphere. The resultant solution was stirred for $5 \mathrm{~min}$ at the same temperature and a solution of $\mathbf{1 6}(25.0 \mathrm{mg}$, $0.0919 \mathrm{mmol})$ in toluene $(0.2 \mathrm{~mL})$ was added. The reaction mixture was stirred for 10 min at the same temperature, quenched with $1 \mathrm{M} \mathrm{HCl}(0.3 \mathrm{~mL})$ and extracted with $\mathrm{Et}_{2} \mathrm{O}$ three times. The combined organic layers were washed with brine, dried over $\mathrm{MgSO}_{4}$, and evaporated under reduced pressure. The resulting residue was purified by column chromatography (hexane-AcOE, 2:1) to afford $17(12.0 \mathrm{mg}, 0.0440 \mathrm{mmol}, 48 \%)$ as a 
colorless solid. Suitable crystals for X-Ray analysis were obtained by steam diffusive atomization (hexane-AcOEt). The crystals obtained were colorless and block-shaped (CCDC 2084101; see details on page S35).

M.p. $173-175^{\circ} \mathrm{C} .[\alpha]^{25}=-183.7\left(\mathrm{c} 0.29, \mathrm{CHCl}_{3}\right)$; IR (KBr); 1730, 1676, 1624, 1276, 1236, 1159, 1041, 965, 918; ${ }^{1} \mathrm{H}$ NMR (400 MHz, $\left.\mathrm{CDCl}_{3}\right) \delta 5.97(1 \mathrm{H}, \mathrm{d}, J=2.3 \mathrm{~Hz})$, $5.04(1 \mathrm{H}, \mathrm{dd}, J=1.4 \mathrm{~Hz}, 1.4 \mathrm{~Hz}), 4.84(1 \mathrm{H}, \mathrm{br}), 2.80-2.60(5 \mathrm{H}, \mathrm{m}), 2.46-2.36(3 \mathrm{H}, \mathrm{m})$, 2.18-2.12 (1H, m), 1.96-1.85 (3H, m), $1.83(3 \mathrm{H}, \mathrm{s}), 1.72-1.65(1 \mathrm{H}, \mathrm{m}), 1.03(3 \mathrm{H}, \mathrm{s}) ;{ }^{13} \mathrm{C}$ $\operatorname{NMR}\left(100 \mathrm{MHz}, \mathrm{CDCl}_{3}\right) \delta 197.8,170.5,155.6,144.7,128.5,116.0,84.2,43.9,39.0$, 33.4, 31.7, 29.2, 26.9, 26.2, 26.0, 23.8, 18.7; HRMS (ESI-TOF) Calcd for $\mathrm{C}_{17} \mathrm{H}_{22} \mathrm{O}_{3} \mathrm{Na}$ $[\mathrm{M}+\mathrm{Na}]^{+}$297.1461. Found 297.1463.

(4aR,5R,9R,11aS)-9-hydroxy-4a-methyl-5,9-di(prop-1-en-2-yl)-4,4a,5,6,7,9,10,11-octah ydrobenzo $[i]$ chromen-2(3H)-one (S5)

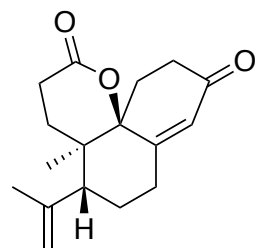

17

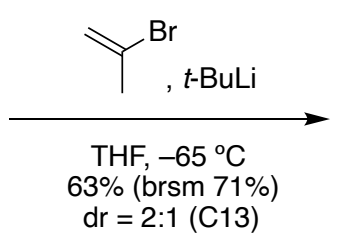

$\mathrm{dr}=2: 1(\mathrm{C} 13)$

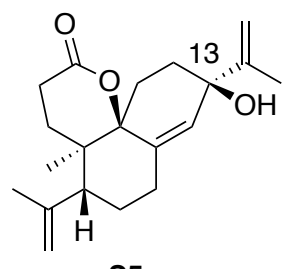

S5

To a stirred solution of $t$ - BuLi $(1.58 \mathrm{M}$ in pentane, $0.140 \mathrm{~mL}, 0.218 \mathrm{mmol})$ in THF $(0.5$ $\mathrm{mL}$ ) was added a solution of 2-bromo-1-propene $(13.0 \mathrm{mg}, 0.109 \mathrm{mmol})$ in THF (0.1 $\mathrm{mL}$ ) at $-78{ }^{\circ} \mathrm{C}$ and stirred for $20 \mathrm{~min}$ at the same temperature. Then a solution of $17(15.0 \mathrm{mg}, 0.0540 \mathrm{mmol})$ in THF $(0.1 \mathrm{~mL})$ was added dropwise at $-65^{\circ} \mathrm{C}$. After stirred for $4 \mathrm{~h}$ at the same temperature, the reaction mixture was quenched with sat. $\mathrm{NH}_{4} \mathrm{Cl}$ aqueous solution and extracted with AcOEt three times. The combined organic layers were washed with brine, dried over $\mathrm{MgSO}_{4}$, and evaporated under reduced pressure. The resulting residue was purified by column chromatography (hexaneAcOEt, 3:1) to afford S5 (11.0 mg, $0.0340 \mathrm{mmol}, 63 \%$ (brsm 71\%)) as an inseparable diastereomeric mixture at $\mathrm{C} 13$ and colorless oil. ${ }^{1} \mathrm{H}$ NMR $\left(400 \mathrm{MHz}, \mathrm{CDCl}_{3}\right) 5.62(1 \mathrm{H}$, s), $4.99(1 \mathrm{H}, \mathrm{s}), 4.95(1 \mathrm{H}, \mathrm{s}), 4.88(1 \mathrm{H}, \mathrm{s}), 4.78(1 \mathrm{H}, \mathrm{s}), 2.70-2.46(4 \mathrm{H}, \mathrm{m}), 2.29-2.24$ $(1 \mathrm{H}, \mathrm{m}), 2.02-1.67(6 \mathrm{H}, \mathrm{m}), 1.60(2 \mathrm{H}, \mathrm{m}), 1.83(3 \mathrm{H}, \mathrm{s}), 1.79(3 \mathrm{H}, \mathrm{s}), 0.92(3 \mathrm{H}, \mathrm{s}) ;{ }^{13} \mathrm{C}$ 
NMR $\left(100 \mathrm{MHz}, \mathrm{CDCl}_{3}\right) \delta 171.6,146.5,145.4,133.1,115.3,113.9,85.4,74.2,44.1$, 38.6, 31.1, 30.3, 29.6, 28.3, 26.9, 26.7, 26.1, 23.9, 18.6, 17.5; HRMS (ESI-TOF) Calcd for $\mathrm{C}_{20} \mathrm{H}_{28} \mathrm{O}_{3} \mathrm{Na}[\mathrm{M}+\mathrm{Na}]^{+} 339.1931$. Found 341.1933.

(4aR,5R,9S,11aS)-9-hydroxy-9-isopropyl-4a-methyl-5-(prop-1-en-2-yl)-4,4a,5,6,7,9,10, 11-octahydrobenzo $[i]$ chromen-2(3H)-one (2)

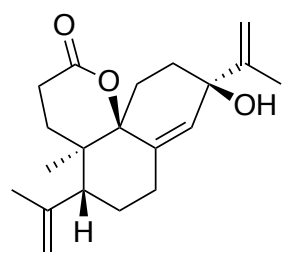

S5

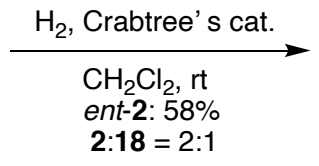

$2: 18=2: 1$

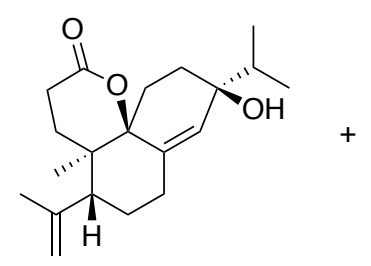

ent-callilongisin B (2)

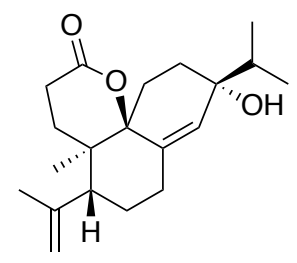

18

To a stirred solution of $\mathbf{S 5}(5.00 \mathrm{mg}, 0.0150 \mathrm{mmol})$ in $\mathrm{CH}_{2} \mathrm{Cl}_{2}(0.2 \mathrm{~mL})$ was added Crabtree's catalyst $(2.50 \mathrm{mg}, 0.00300 \mathrm{mmol})$ under Ar atmosphere at room temperature. After the reaction mixture was stirred under $\mathrm{H}_{2}$ atmosphere for $30 \mathrm{~min}$ at room temperature, the mixture was concentrated in vacuo. The resulting residue was purified by column chromatography (hexane-AcOEt, 3:1) and preparative TLC (hexane-AcOEt, $3: 1)$ three times to afford $2(2.90 \mathrm{mg}, 0.00900 \mathrm{mmol}, 58 \%)$ as a colorless oil and $\mathbf{1 8}$ (1.30 mg, $0.00400 \mathrm{mmol}, 25 \%)$ as a colorless oil.

Data for 2 (ent-callilongisin B)

$[\alpha]^{25}{ }_{\mathrm{D}}=-21.8(\mathrm{c} 0.06, \mathrm{MeOH}), \mathrm{IR} ; 2922,1633,1495,1271,1038,972 ;{ }^{1} \mathrm{H}$ NMR (400 $\left.\mathrm{MHz}, \mathrm{CDCl}_{3}\right) \delta 5.64(1 \mathrm{H}, \mathrm{s}), 4.98(1 \mathrm{H}, \mathrm{s}), 4.79(1 \mathrm{H}, \mathrm{s}), 2.69-2.37(5 \mathrm{H}, \mathrm{m}), 2.22-2.17$ $(1 \mathrm{H}, \mathrm{m}), 1.93-1.69(5 \mathrm{H}, \mathrm{m}), 1.80(3 \mathrm{H}, \mathrm{s}), 1.54(2 \mathrm{H}, \mathrm{m}), 1.01(3 \mathrm{H}, \mathrm{s}), 0.96(3 \mathrm{H}, \mathrm{d}, J=$ $6.8 \mathrm{~Hz}), 0.91(3 \mathrm{H}, \mathrm{d}, J=6.8 \mathrm{~Hz}) ;{ }^{13} \mathrm{C} \mathrm{NMR}\left(125 \mathrm{MHz}, \mathrm{CDCl}_{3}\right) \delta 172.1,145.6,137.2$, $131.3,115.2,85.6,71.3,44.1,38.4,38.0,31.3,27.1,26.9,26.8,26.4,26.3,23.8,18.8$, 17.3, 16.4; HRMS (ESI-TOF) Calcd for $\mathrm{C}_{20} \mathrm{H}_{30} \mathrm{O}_{3} \mathrm{Na}[\mathrm{M}+\mathrm{Na}]^{+} 341.2087$. Found 341.2086 .

Data for 18

$[\alpha]^{25}=-38.1(\mathrm{c} 0.17, \mathrm{MeOH}), \mathrm{IR} ; 2924,1728,1463,1379,1263,1036,971 ;{ }^{1} \mathrm{H}$ NMR $\left(400 \mathrm{MHz}, \mathrm{CDCl}_{3}\right) \delta 5.72(1 \mathrm{H}, \mathrm{s}), 4.99(1 \mathrm{H}, \mathrm{s}), 4.77(1 \mathrm{H}, \mathrm{s}), 2.73-2.46(4 \mathrm{H}, \mathrm{m})$, 
2.20-2.08 (4H, m), 1.88-1.67 (3H, m), $1.79(3 \mathrm{H}, \mathrm{s}), 0.98(3 \mathrm{H}, \mathrm{d}, J=6.9), 0.95(3 \mathrm{H}, \mathrm{s})$, $0.93(3 \mathrm{H}, \mathrm{d}, J=6.9),{ }^{13} \mathrm{C} \mathrm{NMR}\left(100 \mathrm{MHz}, \mathrm{CDCl}_{3}\right) \delta 171.8,145.5,134.1,115.4,85.6$, 71.8, 44.4, 38.9, 34.9, 31.4, 30.1, 29.7, 28.7, 27.5, 26.8, 26.3, 24.1, 18.7, 17.1, 16.8; HRMS (ESI-TOF) Calcd for $\mathrm{C}_{20} \mathrm{H}_{30} \mathrm{O}_{3} \mathrm{Na}[\mathrm{M}+\mathrm{Na}]^{+}$341.2087. Found 341.2090.

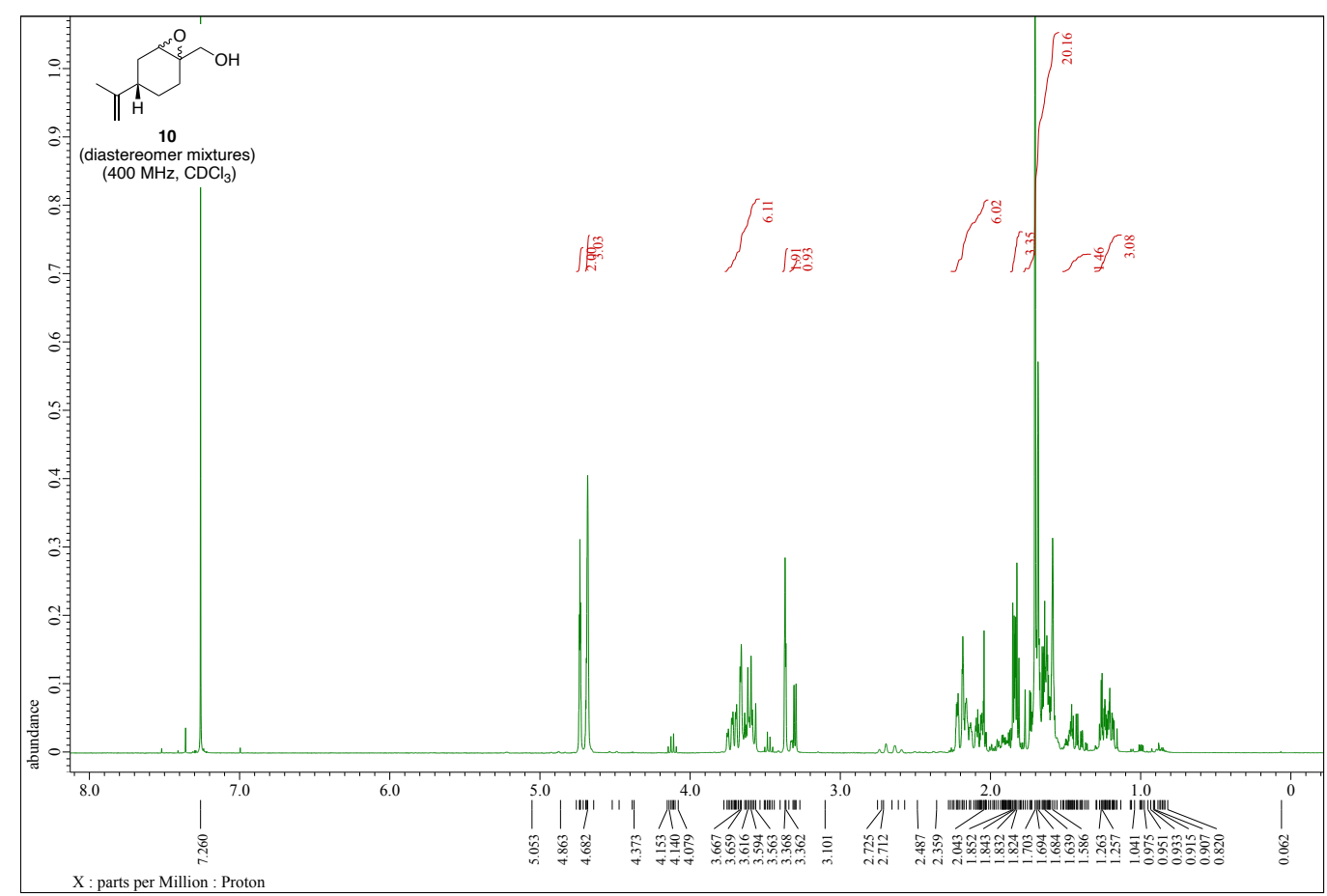



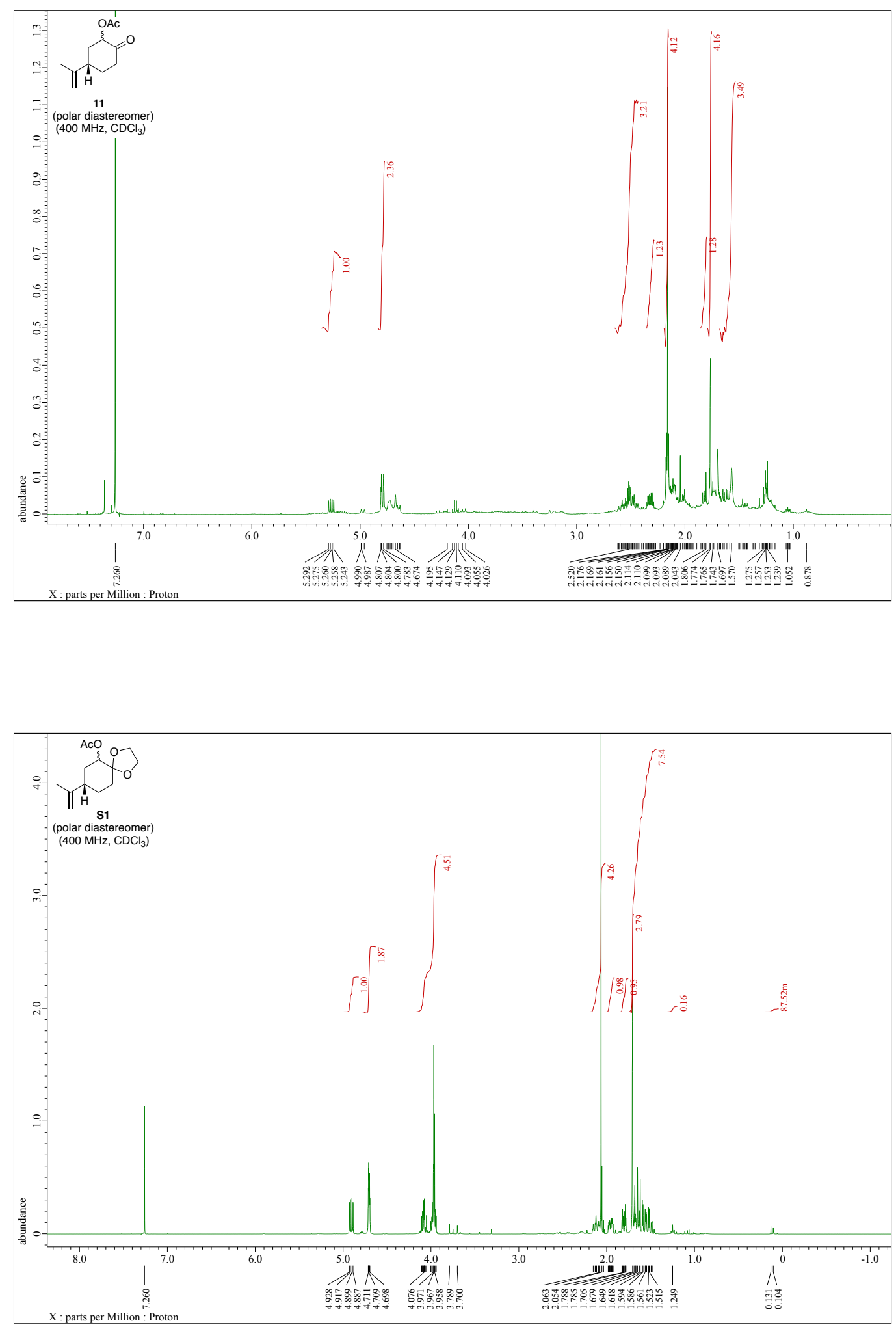

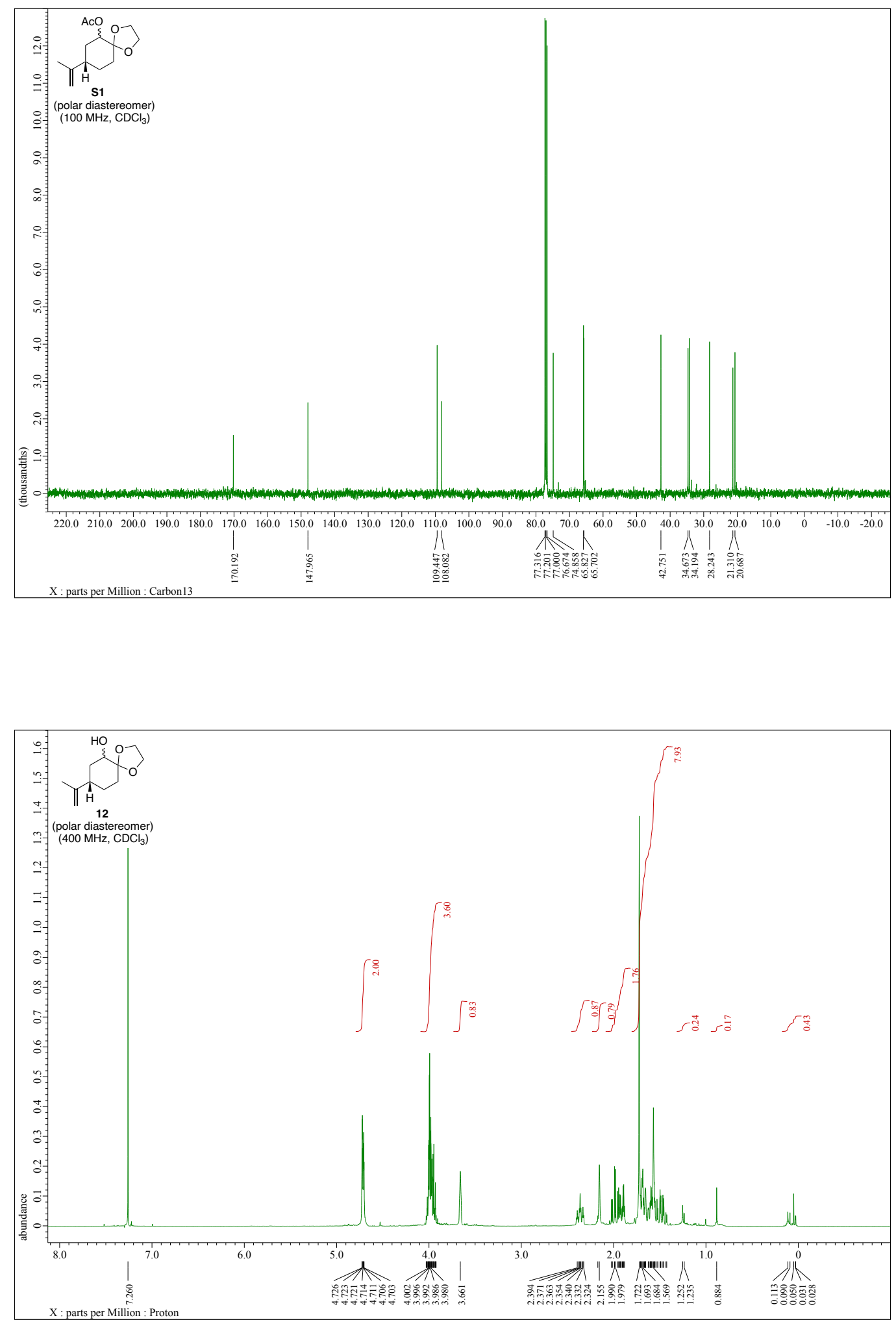

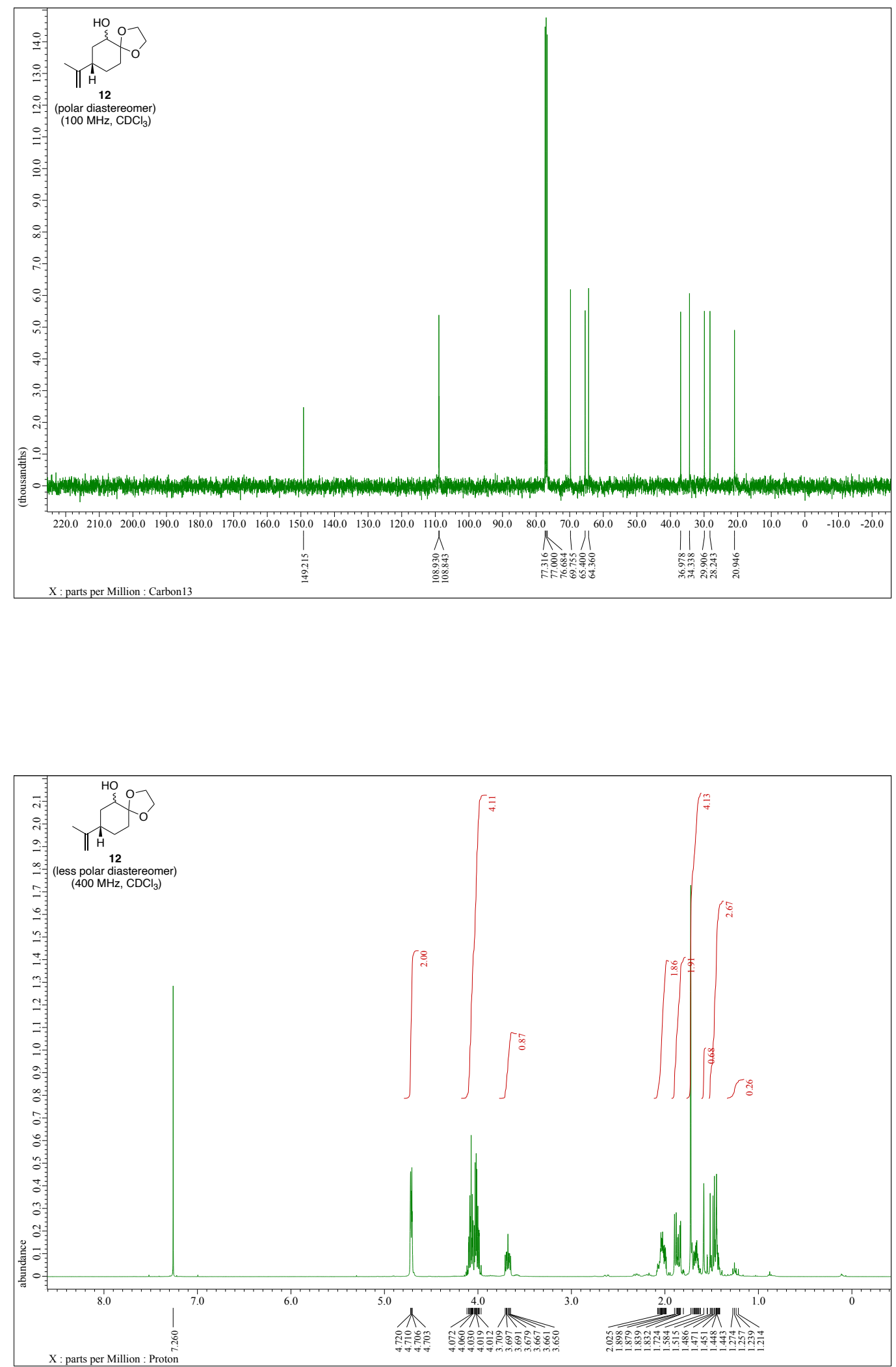

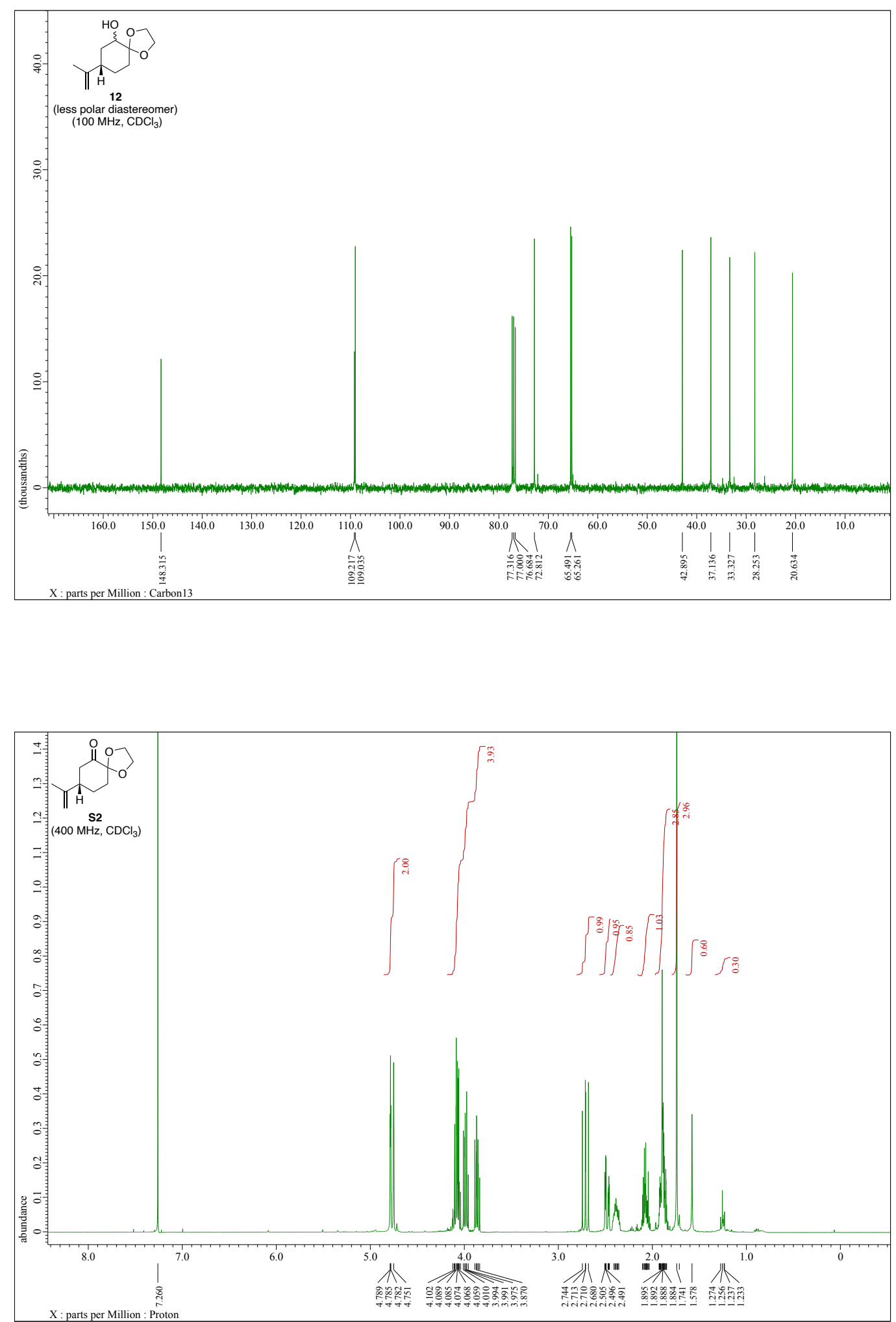

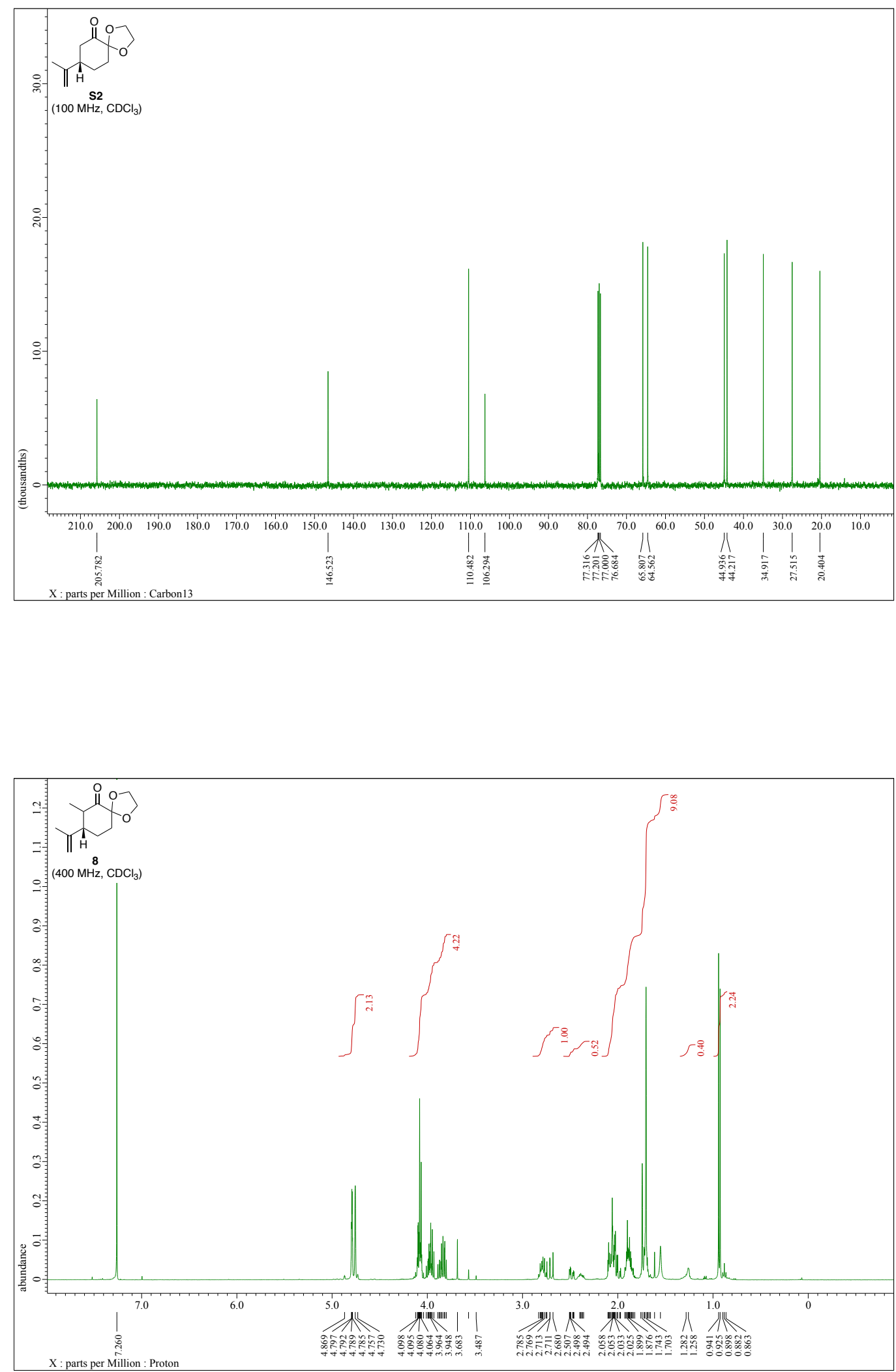

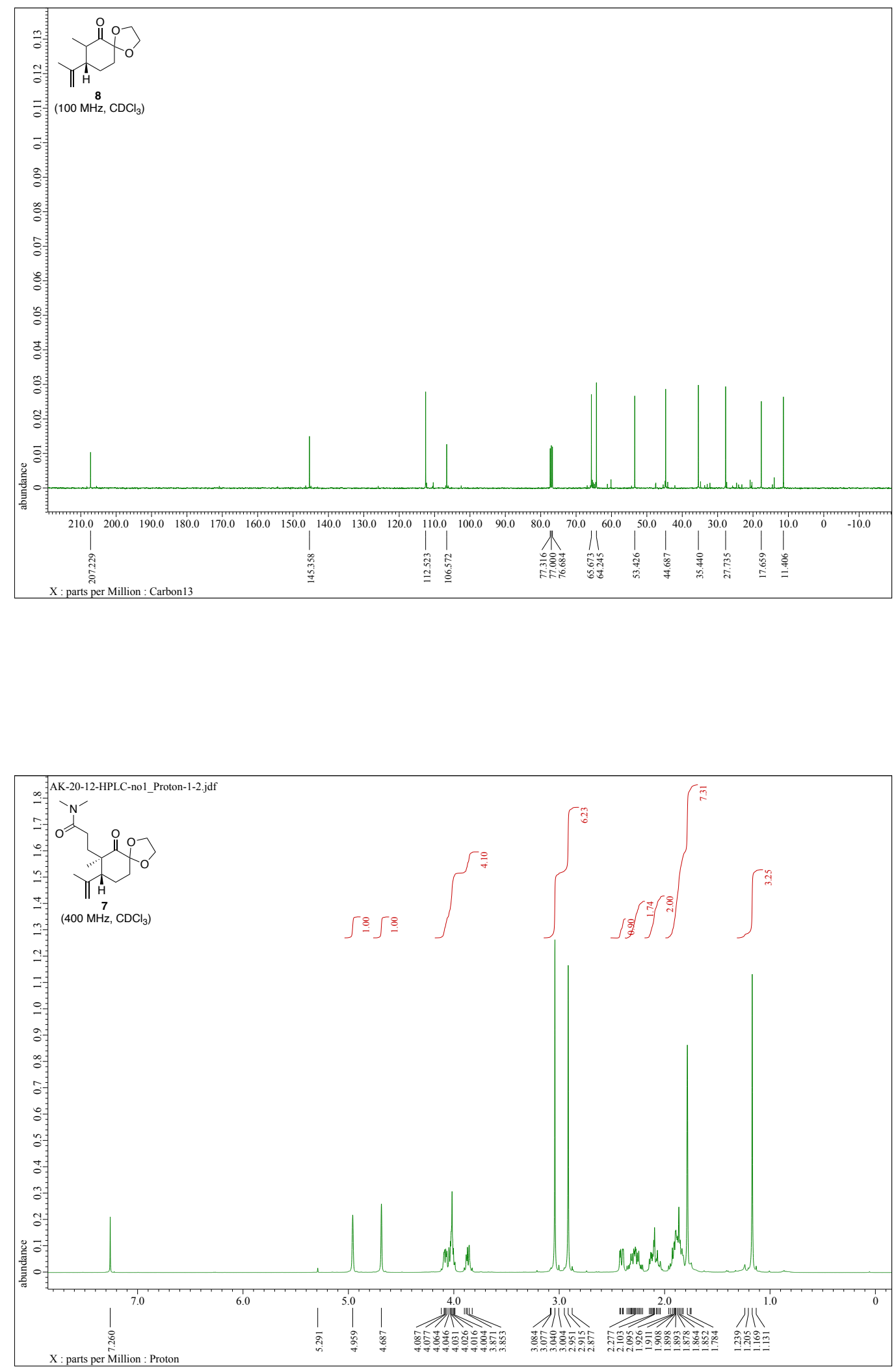

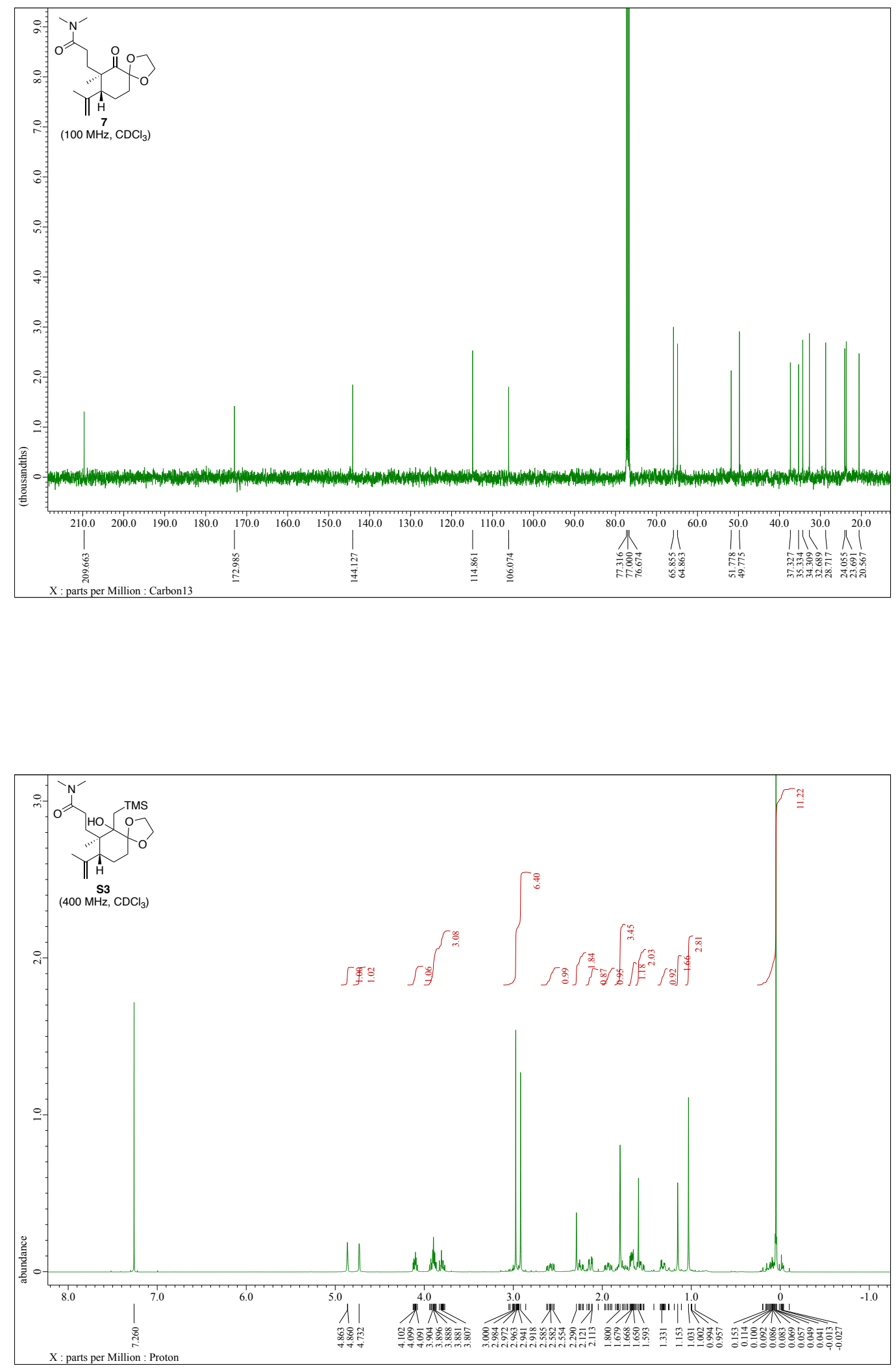

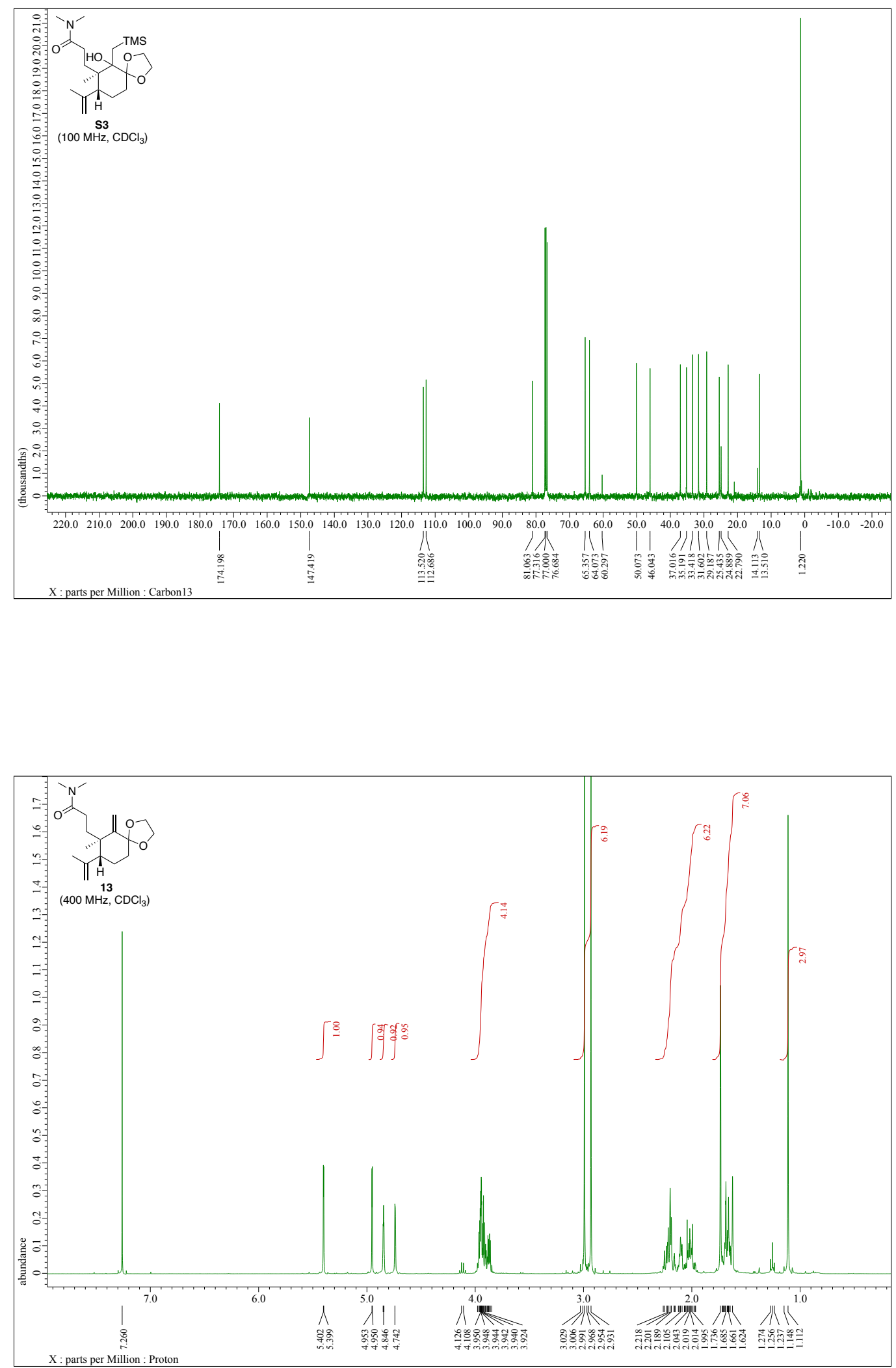

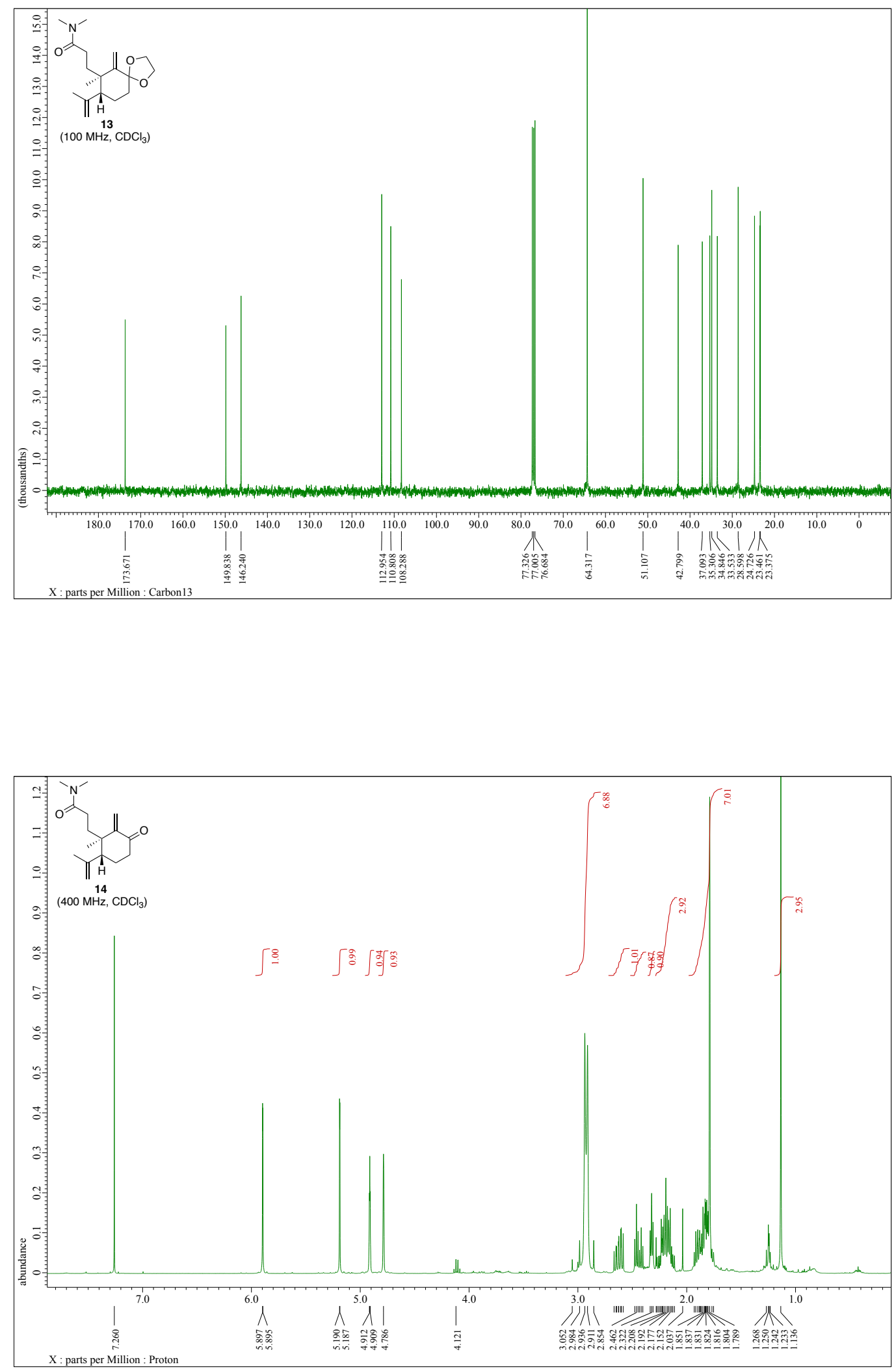

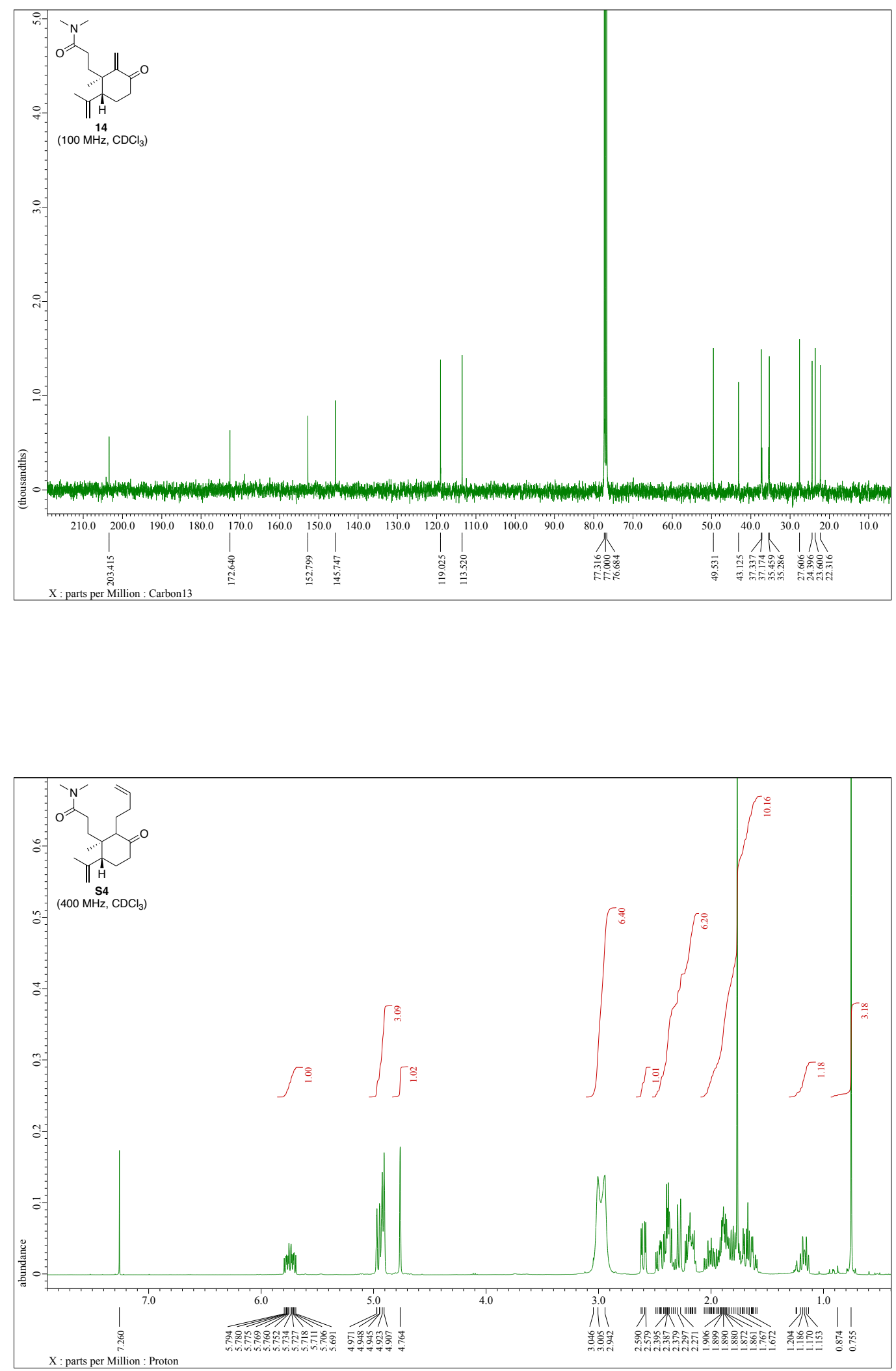

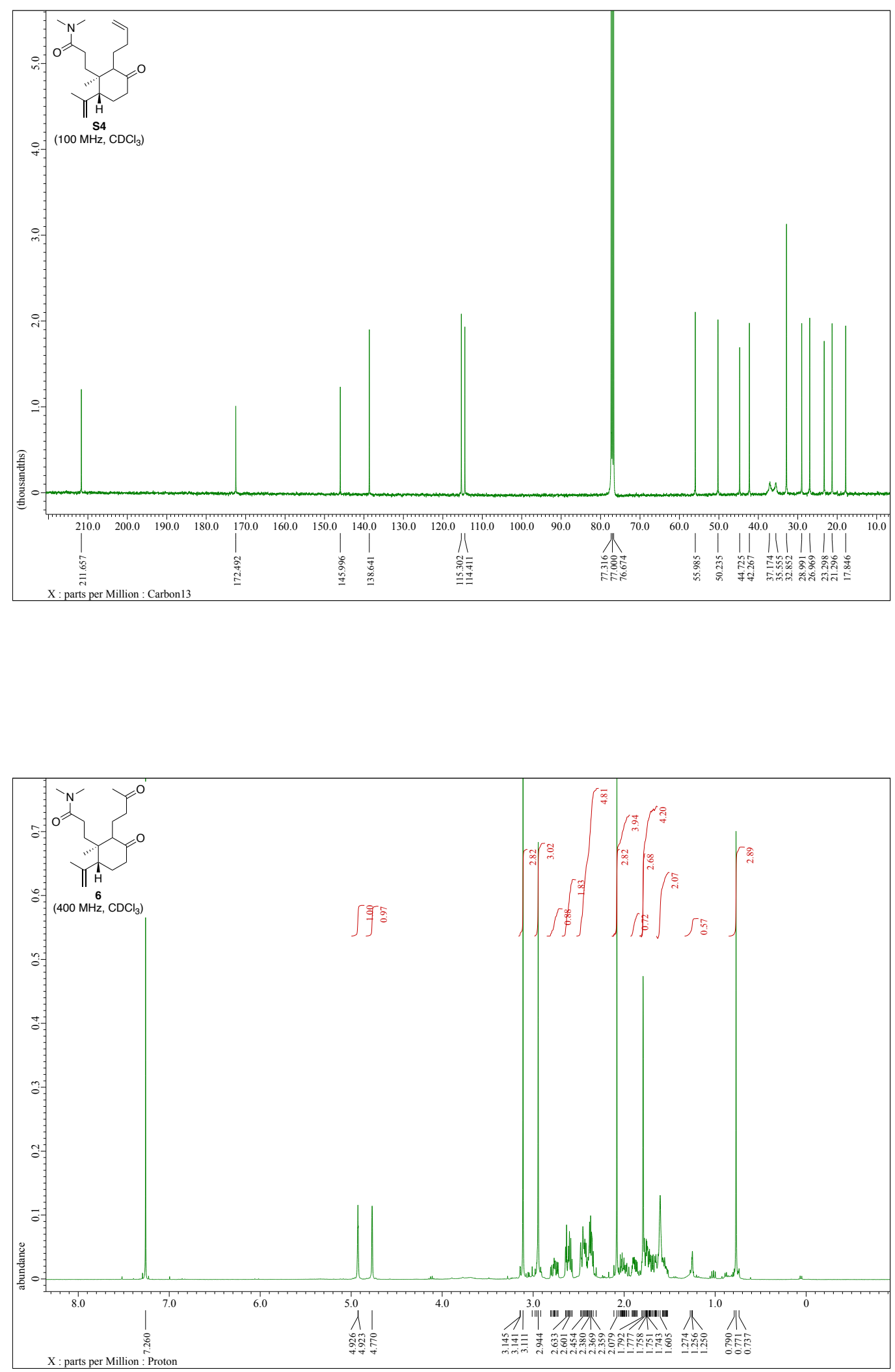

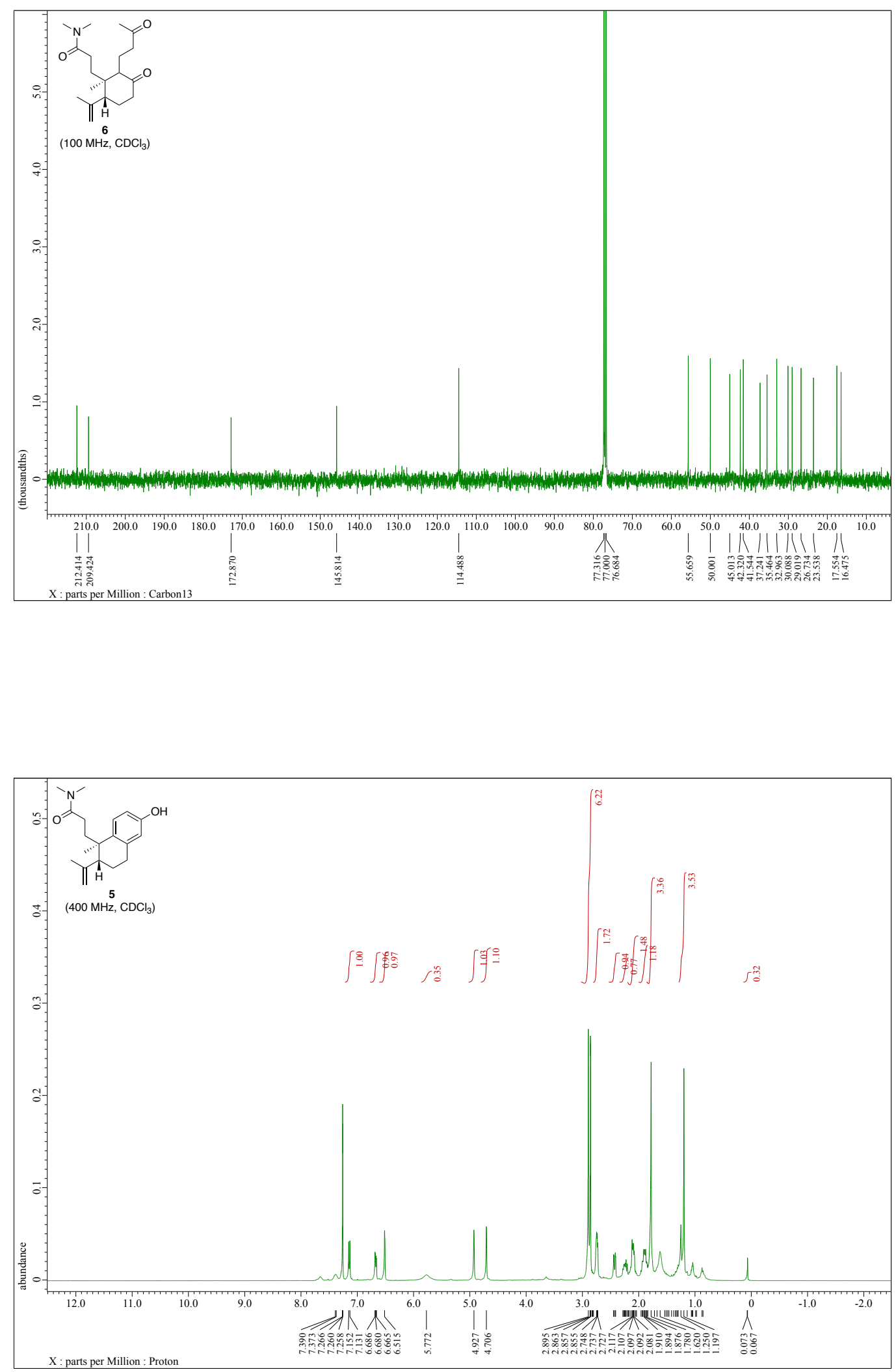

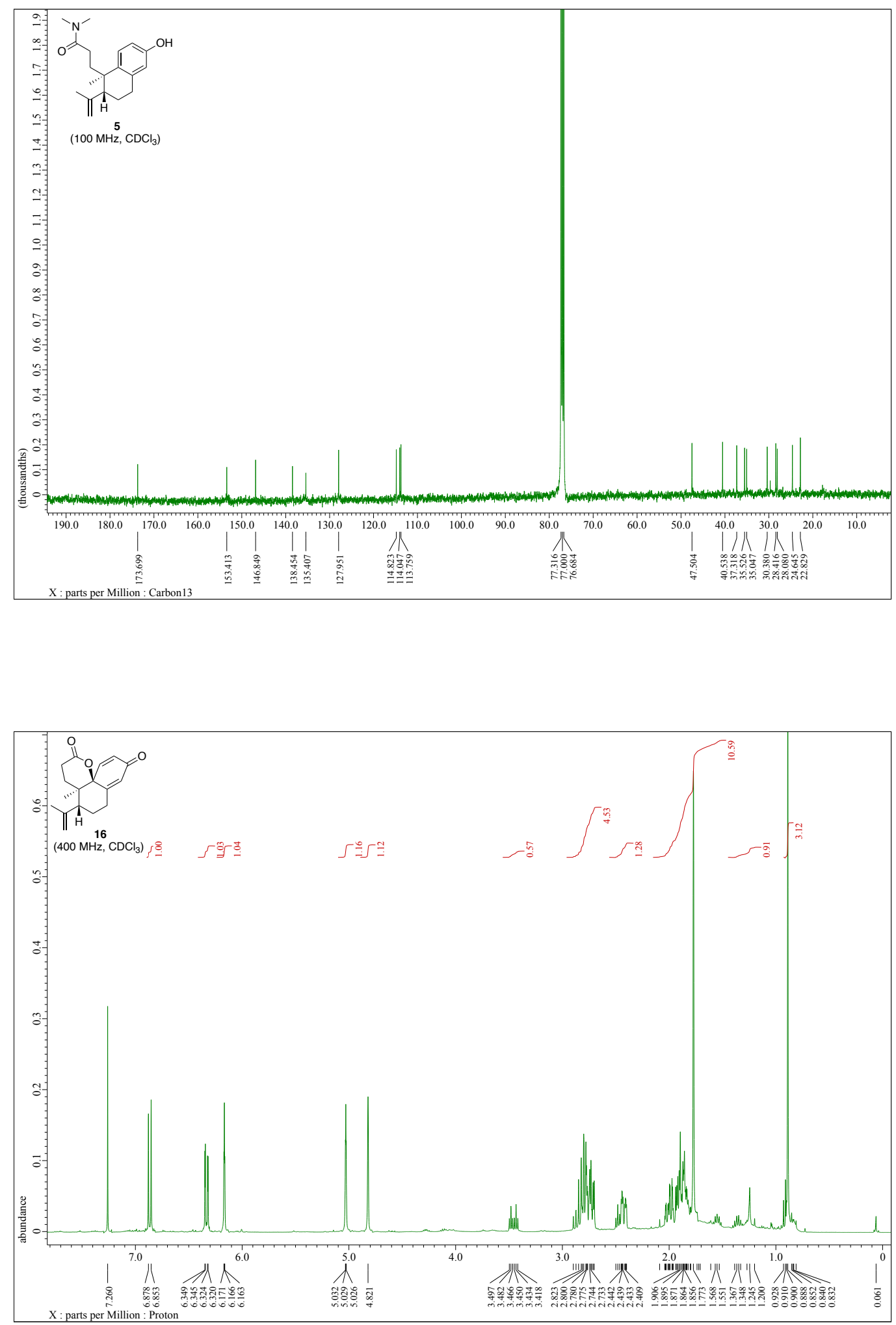

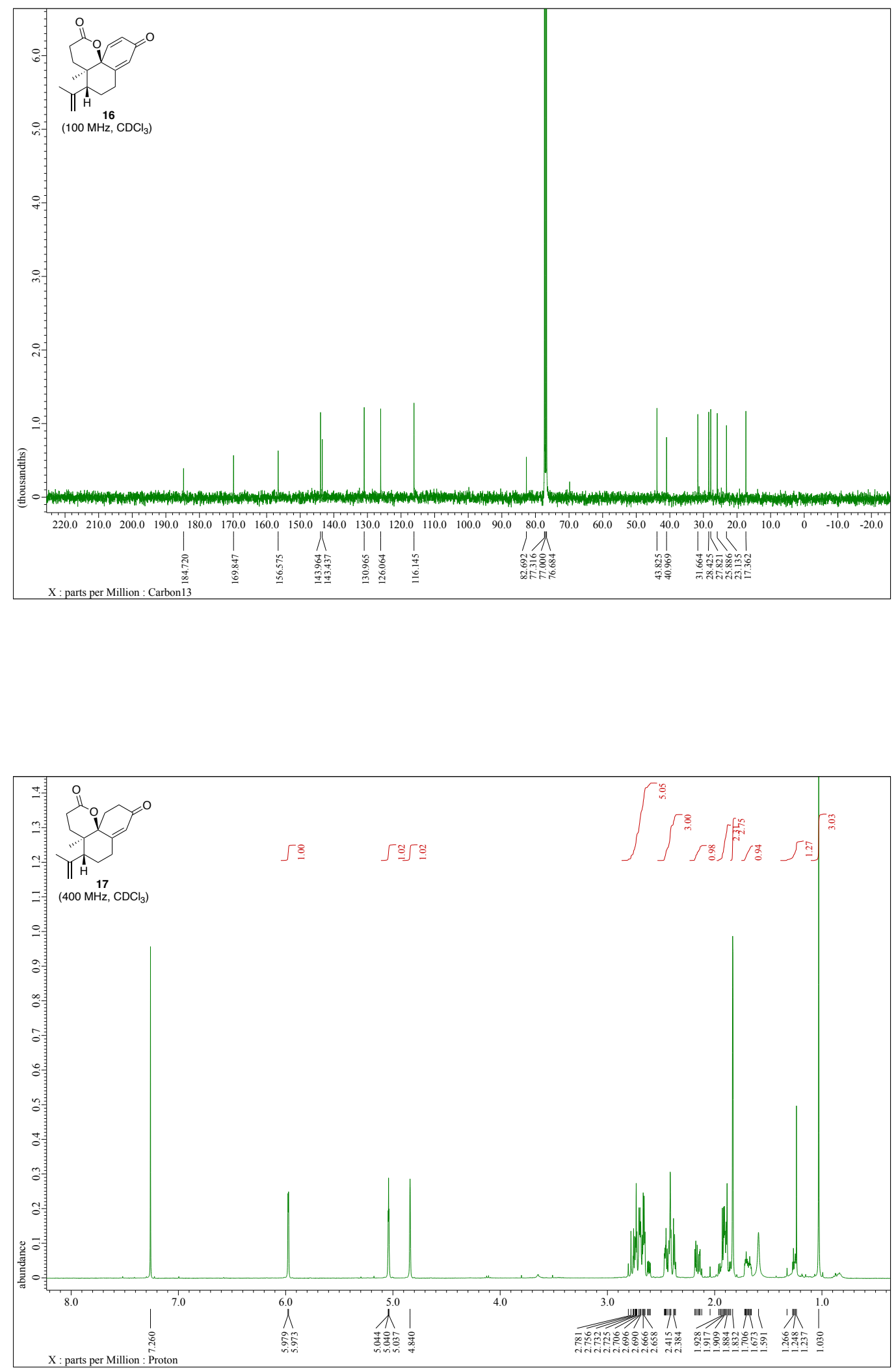

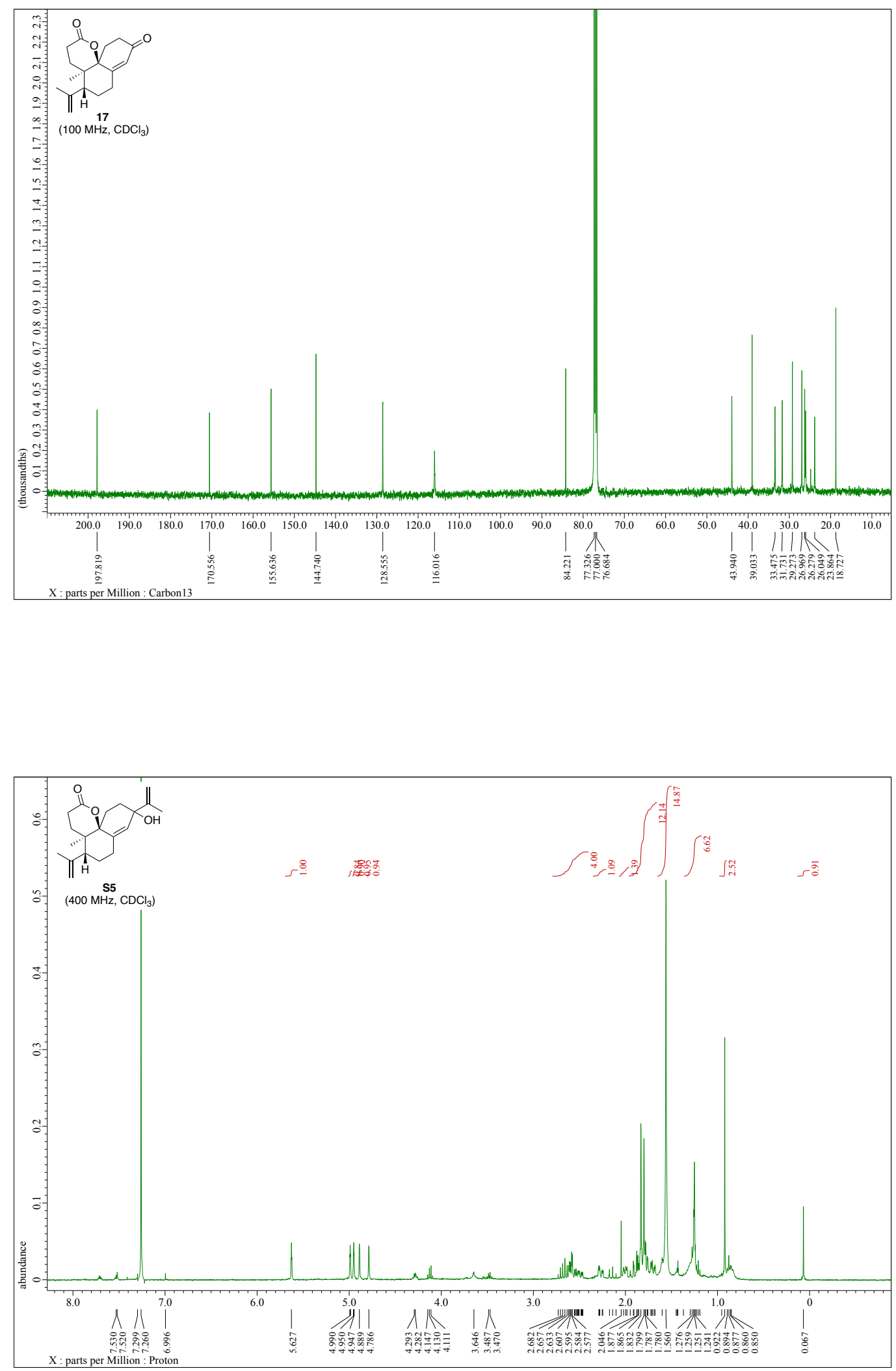


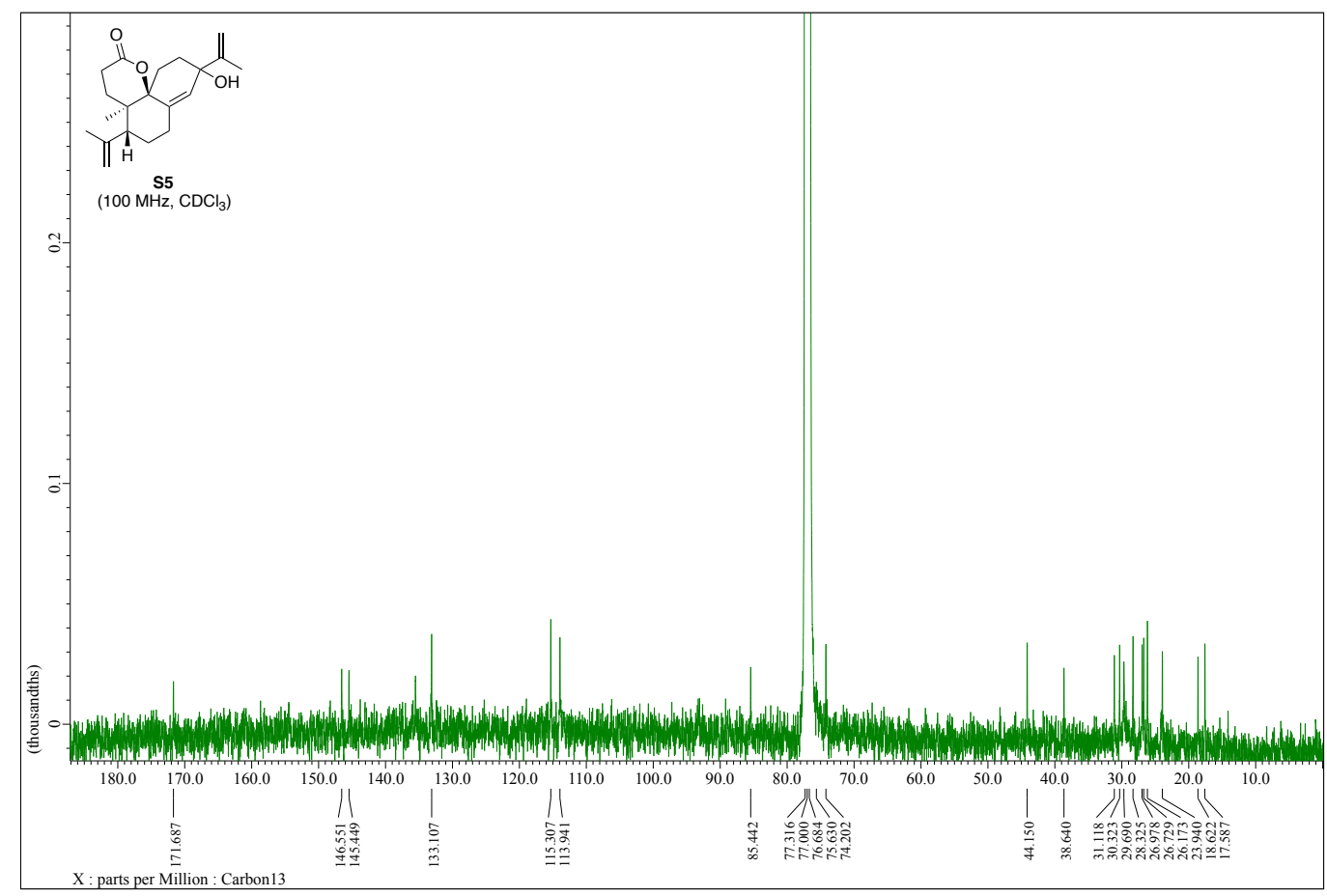

upper: synthetic, bottom: literature for ${ }^{1} \mathrm{H}$ NMR 

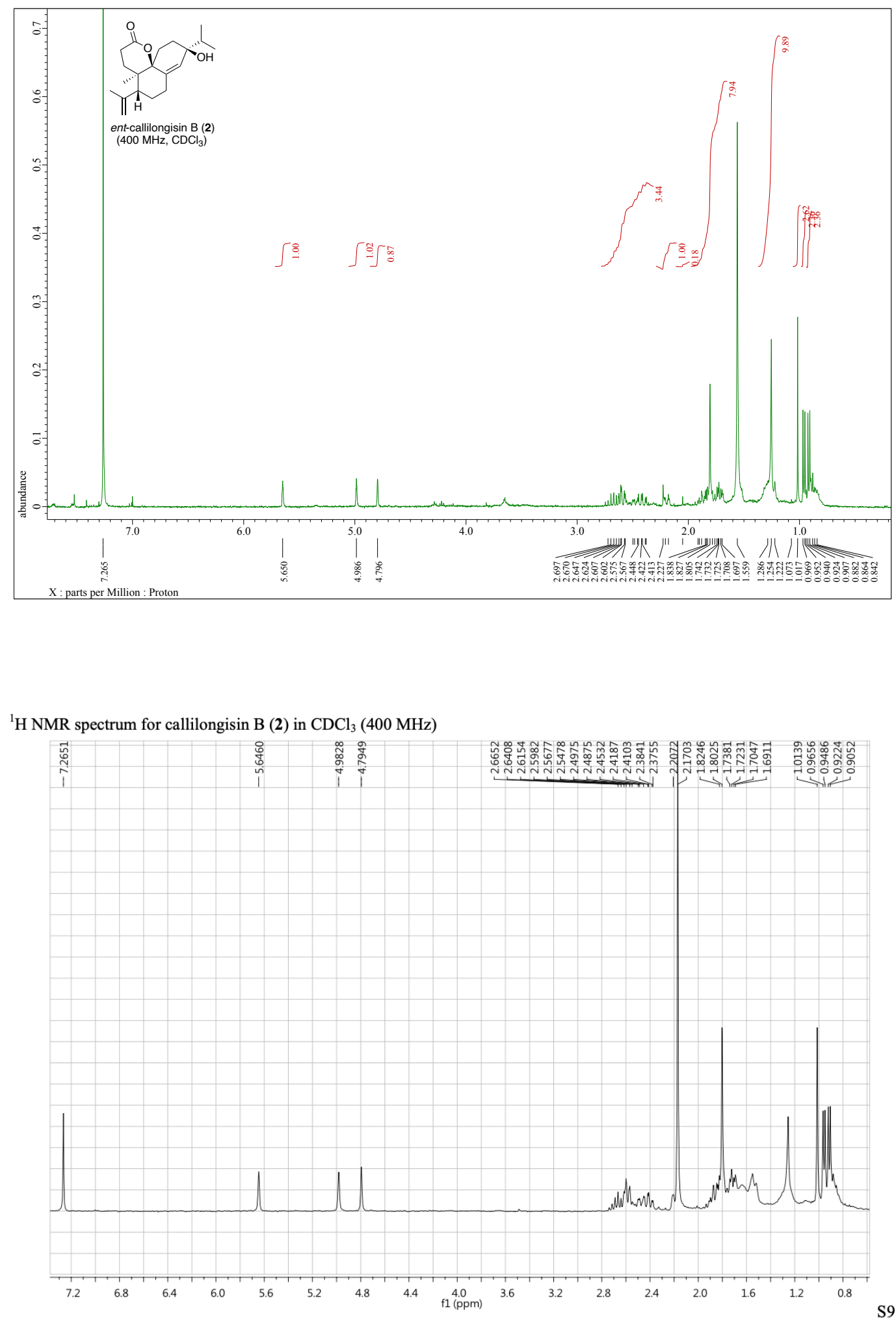

upper: synthetic, bottom: literature for ${ }^{13} \mathrm{C}$ NMR 

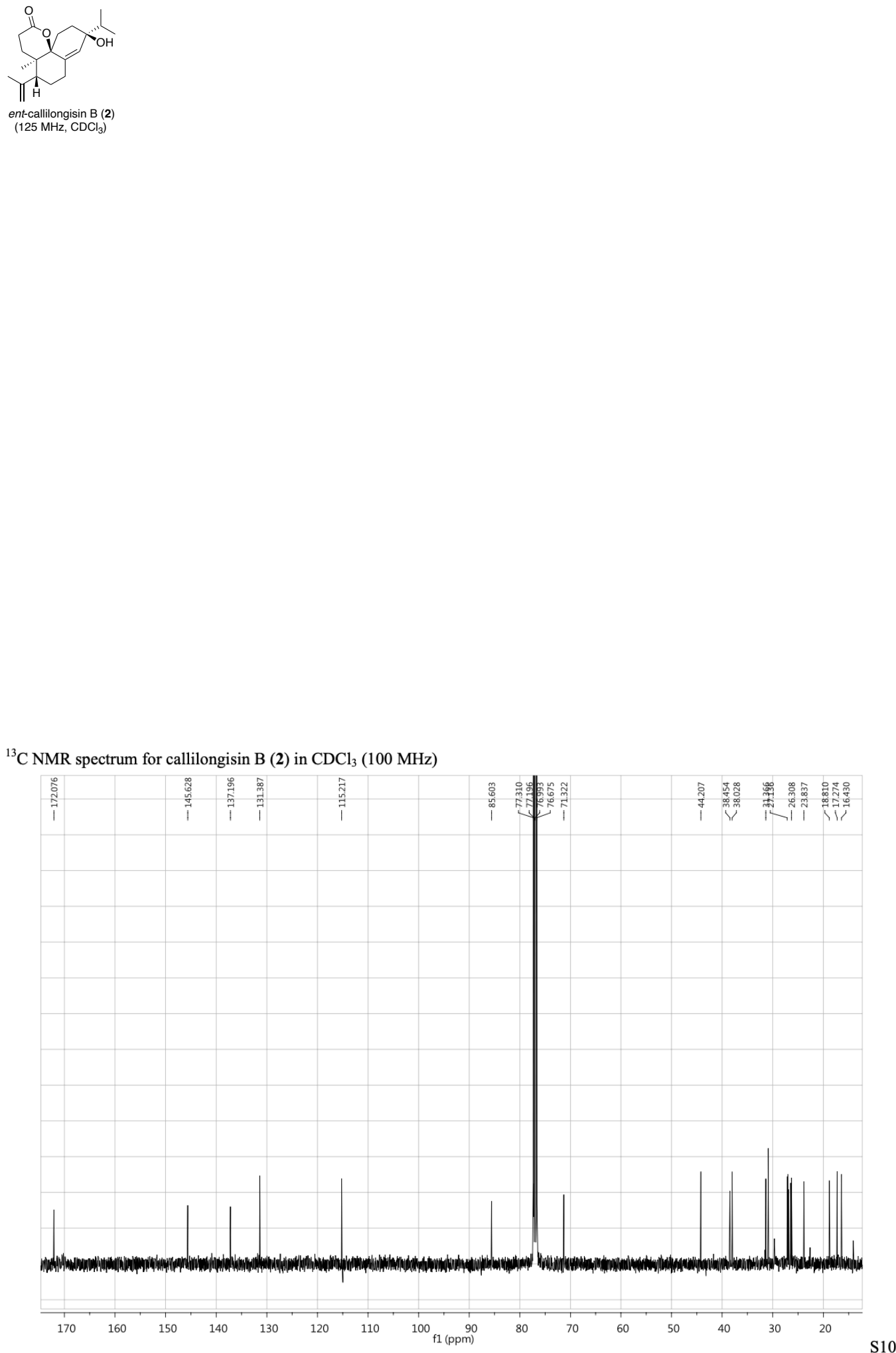

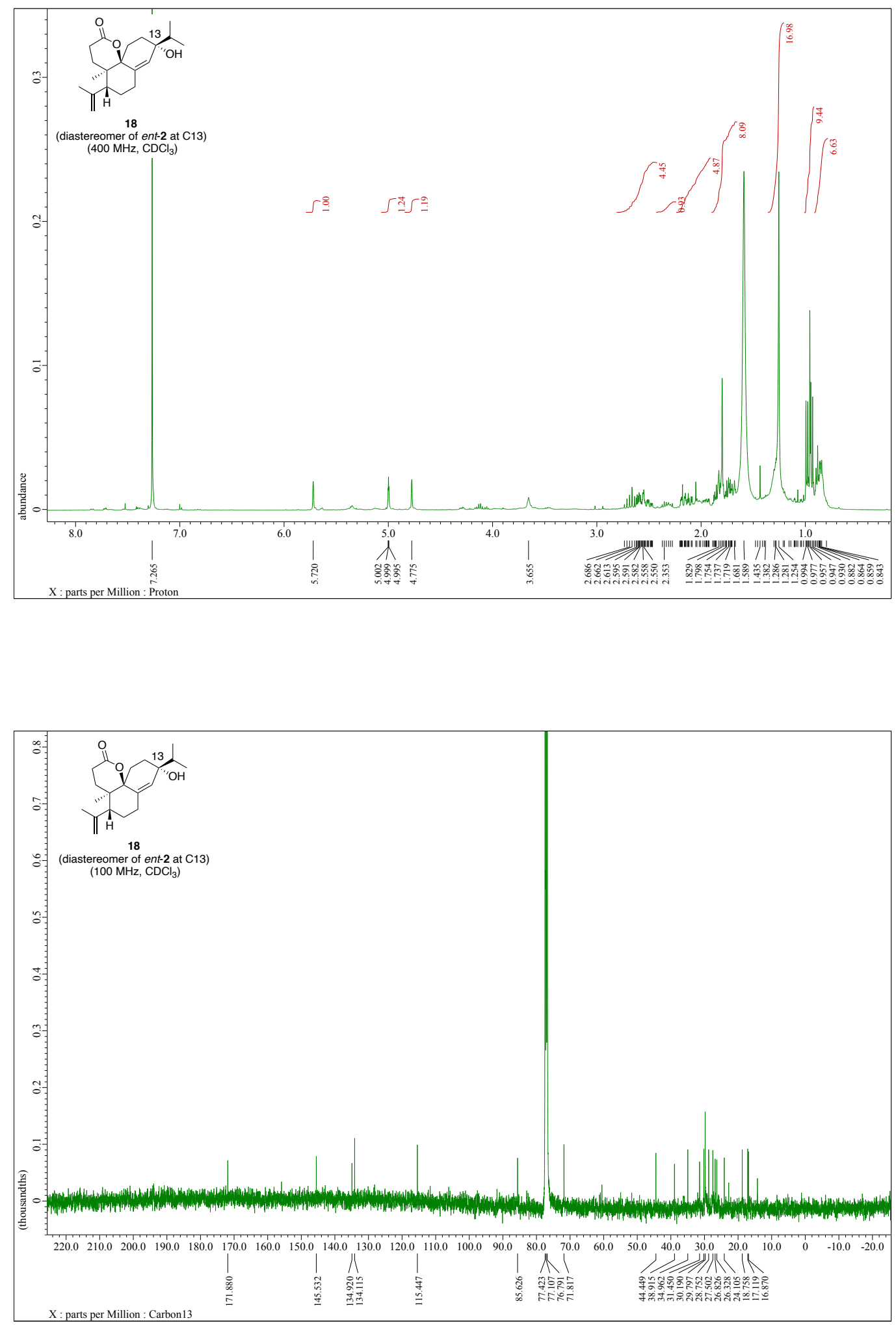
X-ray single-crystal diffraction data (relative configuration of 17) : CCDC 2084101

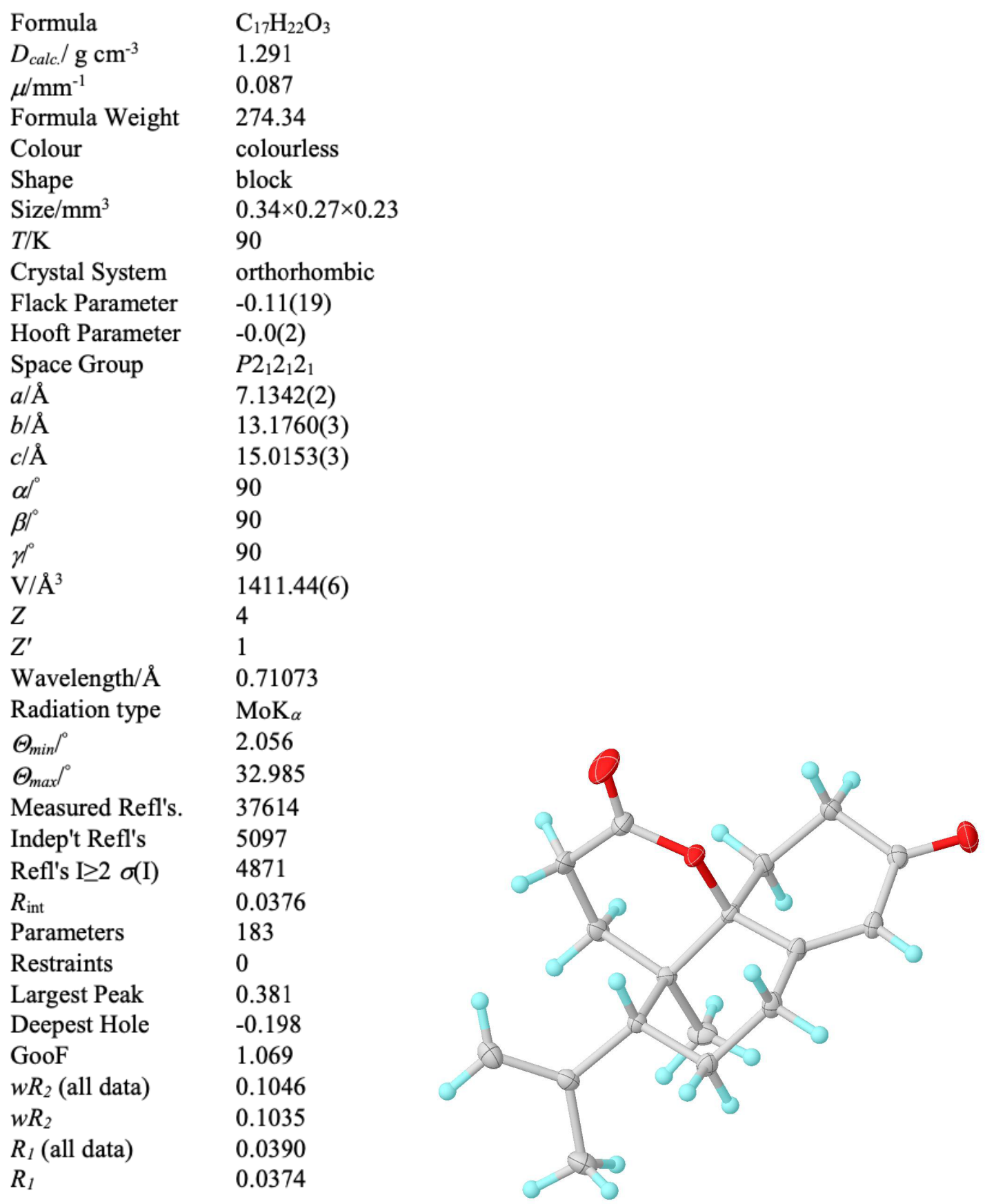

